\title{
RESEARCH
}

Open Access

\section{Upregulation of RIN3 induces endosomal dysfunction in Alzheimer's disease}

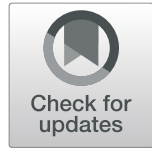

Ruinan Shen ${ }^{1,2}$, Xiaobei Zhao ${ }^{2}$, Lu He ${ }^{1}$, Yongbo Ding ${ }^{3}$, Wei Xu ${ }^{1,2}$, Suzhen Lin ${ }^{1,2}$, Savannah Fang ${ }^{2}$, Wanlin Yang ${ }^{1,2,4}$, Kijung Sung ${ }^{2}$, Brian Spencer ${ }^{2}$, Robert A. Rissman ${ }^{2,5}$, Ming Lei ${ }^{3}$, Jianqing Ding ${ }^{1 *}$ and Chengbiao $\mathrm{Wu}^{2^{*}}$ (D)

\begin{abstract}
Background: In Alzheimer's Disease (AD), about one-third of the risk genes identified by GWAS encode proteins that function predominantly in the endocytic pathways. Among them, the Ras and Rab Interactor 3(RIN3) is a guanine nucleotide exchange factor (GEF) for the Rab5 small GTPase family and has been implicated to be a risk factor for both late onset $A D(L O A D)$ and sporadic early onset AD (sEOAD). However, how RIN3 is linked to AD pathogenesis is currently undefined.
\end{abstract}

Methods: Quantitative PCR and immunoblotting were used to measure the RIN3 expression level in mouse brain tissues and cultured basal forebrain cholinergic neuron (BFCNs). Immunostaining was used to define subcellular localization of RIN3 and to visualize endosomal changes in cultured primary BFCNs and PC12 cells. Recombinant flag-tagged RIN3 protein was purified from HEK293T cells and was used to define RIN3-interactomes by mass spectrometry. RIN3-interacting partners were validated by co-immunoprecipitation, immunofluorescence and yeast two hybrid assays. Live imaging of primary neurons was used to examine axonal transport of amyloid precursor protein (APP) and $\beta$-secretase 1 (BACE1). Immunoblotting was used to detect protein expression, processing of APP and phosphorylated forms of Tau.

Results: We have shown that RIN3 mRNA level was significantly increased in the hippocampus and cortex of APP/PS1 mouse brain. Basal forebrain cholinergic neurons (BFCNs) cultured from E18 APP/PS1 mouse embryos also showed increased RIN3 expression accompanied by early endosome enlargement. In addition, via its proline rich domain, RIN3 recruited BIN1 (bridging integrator 1) and CD2AP (CD2 associated protein), two other AD risk factors, to early endosomes. Interestingly, overexpression of RIN3 or CD2AP promoted APP cleavage to increase its carboxyl terminal fragments (CTFs) in PC12 cells. Upregulation of RIN3 or the neuronal isoform of BIN1 increased phosphorylated Tau level. Therefore, upregulation of RIN3 expression promoted accumulation of APP CTFs and increased phosphorylated Tau. These effects by RIN3 was rescued by the expression of a dominant negative Rab5 (Rab5 ${ }^{\mathrm{S} 34 \mathrm{~N}}$ ) construct. Our study has thus pointed to that RIN3 acts through Rab5 to impact endosomal trafficking and signaling.

(Continued on next page)

\footnotetext{
*Correspondence: jqding18@123.com; chw049@ucsd.edu

'Institute of Neurology, Ruijing Hospital, Shanghai JiaoTong University School of Medicine, 197 Ruijin Er Rd., Shanghai 200025, China

${ }^{2}$ Department of Neurosciences, University of California San Diego School of Medicine, Room 312 MC-0624,9500 Gilman Drive, La Jolla, CA 92093-0624, USA

Full list of author information is available at the end of the article
}

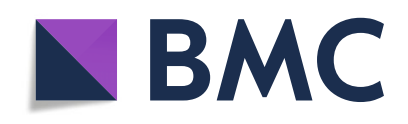

(- The Author(s). 2020 Open Access This article is licensed under a Creative Commons Attribution 4.0 International License, which permits use, sharing, adaptation, distribution and reproduction in any medium or format, as long as you give appropriate credit to the original author(s) and the source, provide a link to the Creative Commons licence, and indicate if changes were made. The images or other third party material in this article are included in the article's Creative Commons licence, unless indicated otherwise in a credit line to the material. If material is not included in the article's Creative Commons licence and your intended use is not permitted by statutory regulation or exceeds the permitted use, you will need to obtain permission directly from the copyright holder. To view a copy of this licence, visit http://creativecommons.org/licenses/by/4.0/. The Creative Commons Public Domain Dedication waiver (http://creativecommons.org/publicdomain/zero/1.0/) applies to the data made available in this article, unless otherwise stated in a credit line to the data. 


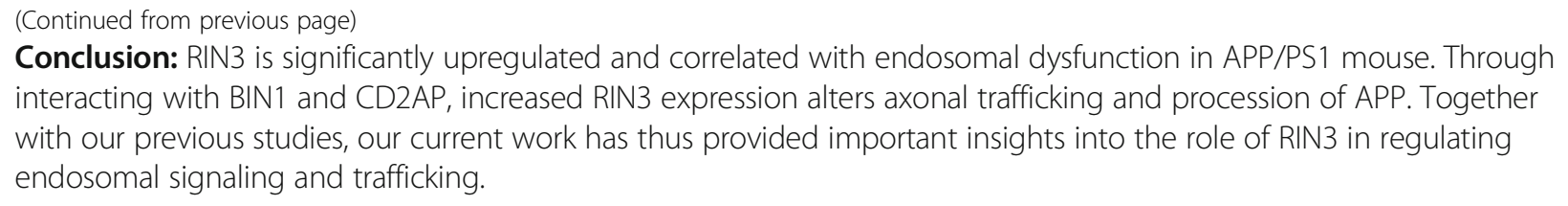

Keywords: Alzheimer's disease (AD), AD risk factors, Endosomes, Trafficking, RIN3, BIN1, CD2AP, Tau

\section{Background}

Alzheimer's disease (AD) is a progressive neurodegenerative disorder that results in memory loss and cognitive impairment [1]. The classical neuropathological hallmarks for $\mathrm{AD}$ include $\mathrm{A} \beta$-amyloid-containing neurotic plaques and phosphorylated Tau-containing neurofibrillary tangles (NFT) [2]. In addition, significant synaptic loss, selective neuronal death, neurotransmitter loss and neuroinflammation are also associated with AD pathology [3, 4]. Despite of significant efforts and investments, there are currently no disease-modifying treatments for $\mathrm{AD}$ [5-7].

One of the most important lessons learned from these failed clinical efforts is that the cause(s) for $\mathrm{AD}$ is far more complex than we have realized. In 2009, apolipoprotein $\mathrm{E}(A P O E)$ that functions in lipid transport, $\mathrm{A} \beta$ trafficking, synaptic function, immune regulation, and intracellular signaling [8], was identified as a risk factor for LOAD [9]. More recently, large scale GWAS and a meta-analysis of LOAD have identified more than 20 additional risk factors [10-13]. Identification of these additional risk factors further illustrates the extraordinary genetic complexity of $\mathrm{AD}$.

A significant number of these newly identified loci (PICALM, BIN1, EPHA1, CD2AP, MEF2C, PTK2B, SORL1 and RIN3) that encode products all function significantly in endocytic trafficking and signaling [4]. Given that neurons have the most extraordinary architecture with elaborate dendrites/ axons and will undoubtedly impose a significant need for endocytic processes, it is not surprising that these early endocytic pathways are found to be dysregulated in AD [14-17]. For instance, amyloidogenic cleavage of APP to yield A $\beta$ likely occurs predominantly in the intracellular compartments. Under normal conditions, early endosome, marked by Rab5, is a major site of APP processing by $\beta$-secretase (BACE1) to yield the $\beta$-cleavage $C$-terminal fragment $(\beta C T F)$, which is further processed in late endosomes/trans-Golgi network (TGN) to give rise to $A \beta[17-21]$. Therefore, trafficking and processing of APP to produce toxic $\beta C T F s / A \beta$ is intimately regulated by the endocytic pathways.

Although much less is known about the role of RIN3, increasing evidence has pointed to that RIN3 plays an important role in AD. Initial GWAS studies identified a locus (rs10498633, G/T), upstream of the RIN3 coding sequences within its enhancer region. It is speculated that this single nucleotide polymorphism (SNP) likely results in increased expression of RIN3 in AD [11]. When comparing extreme AD cases with centenarian controls, the variant effect size for the SLC24A4/RIN3 SNP increased by 4.5 -fold, versus 2-folds for APOE- 84 and 6.5folds for TREM2 (R74H) variant in comparison to published effect sizes [22]. A more recent case-control study of whole-exome sequencing of $93 \mathrm{sEOAD}$ patients has discovered a missense variant $(r s 150221413, \mathrm{G} / \mathrm{T})$ that results in a substitution of $63 \mathrm{~W}>\mathrm{C}$ the $\mathrm{SH} 2$ (Src Homology 2) of RIN3 [23]. The RIN3 ${ }^{\mathrm{W} 63 \mathrm{C}}$ variant is likely associated with sEOAD (OR, 4.56; 95\% CI, 1.26-16.48; $P=.02, \mathrm{BP}=0.091$ ) [23]. Furthermore, in a 2017 study of gene methylation profiling of blood and brain samples from $22 \mathrm{AD}$ and 26 normal control subjects $(27$ males, 21 females), AD samples showed significant group-wide hypomethylation of $7 \mathrm{CpGs}$ located within the 3'UTR of RIN3 (CpG1 $\mathrm{p}=0.019$, CpG2 $\mathrm{p}=0.018$, CpG3 $\mathrm{p}=$ 0.012, CpG4 $\mathrm{p}=0.009$, CpG5 $\mathrm{p}=0.002$, CpG6 $\mathrm{p}=$ 0.018, and CpG7 $\mathrm{p}=0.013$, respectively) [24]. The effect was specific for RIN3 since other LOAD risk factors (PTK2 $\beta$, ABCA7, SIRT1, or MEF2C) did not show significant changes in methylation of their promoters. Additionally, a genome wide methylation study of mild cognitive impaired Mexican Americans uncovered significant hypo-methylation in RIN3 and three other genes [25]. Together, these studies have suggested the possibility that increased expression of wildtype RIN3 or expression of the RIN3 variant (W63C) may contribute to $\mathrm{AD}$ pathogenesis. However, the underlying mechanism(s) is presently unknown.

RIN3, a guanidine nucleotide exchange factor (GEF), functions as the stimulator and stabilizer for selective members of the Rab5 family (Rab5, 21, 22, 24 and 31) [26, 27]. By cycling between a GDP- bound inactive and a GTP-bound active form, Rab5 regulates endocytosis, intracellular vesicular trafficking [28-30]. However, there is practically nothing known as how increased expression of RIN3 in AD acts on Rab5 to contribute to cellular pathogenesis in $\mathrm{AD}$. We envision that increased expression of RIN3 results in hyperactivation of Rab5 and impairment of endocytic trafficking and signaling. As such, production and accumulation of toxic APP-CTFs is elevated and phosphorylated Tau is increased, thus contributing to neuronal degeneration in AD. Guided by this 
hypothesis, our current study has demonstrated that RIN3 expression was significantly increased in neurons of APP/ PS1 mice; RIN3 interacted with BIN1/CD2AP to regulate APP trafficking and processing; Increased levels of RIN3 significantly impacted endocytic trafficking process and APP cleavage, that may lead to neuronal degeneration in early AD pathogenesis.

\section{Materials and methods}

\section{Ethical statements}

All experiments involving the use of laboratory animals have been approved by the Institutional Animal Care and Use Committees of Shanghai JiaoTong University and University of California San Diego. Surgical and animal procedures were carried out strictly following the NIH Guide for the Care and Use of Laboratory Animals. When possible, every effort was made in our experiments to measure differences between male and female animals.

\section{Animals}

APP/PS1 transgenic animals were obtained from The Jackson Laboratory (number 005864) on the C57BL/6 background. All mice were housed under standard conditions at $22{ }^{\circ} \mathrm{C}$ and a $12 \mathrm{~h}$ light: dark cycle with free access to food and water. Animal care and handling was performed according to the Declaration of Helsinki and approved by the local ethical committees. Both transgenic APP/PS1 and WT mice in the same littermates were analyzed. For mRNA and protein assays of brain tissues, only male mice of different ages $(0.25,1,3,6,9$, 10 months) were used. At least 4 mice in each group.

\section{Quantitative PCR}

Total RNAs from either mouse tissues or cultured neurons were extracted using the Trizol method according to the manufacturer's protocol. The RNA samples were reverse-transcribed into cDNA using the PrimeScript first Strand cDNA Synthesis Kit (Takara). Quantitative real-time PCR method was used to detect the expression of RIN3. The experimental reaction consisted of $40 \mathrm{cy}-$ cles of $95^{\circ} \mathrm{C}$ for $30 \mathrm{~s}, 60^{\circ} \mathrm{C}$ for $5 \mathrm{~s}$, and $72{ }^{\circ} \mathrm{C} 30 \mathrm{~s}$, the PCR products of the target gene and the internal control gene (GAPDH) were detected and quantitated by fluorescent SYBR Green (Takara) using an ABI7500 real-time PCR machine. Threshold cycle values $(\mathrm{Ct})$ were generated at each cycle during a run. Relative quantification of mRNA expression was calculated by the $\Delta \mathrm{Ct}$ method after adjusting the levels to the corresponding internal GAPDH and normalized against WT samples.

\section{Chemicals, reagents, and antibodies}

HBSS, GlutaMAX, Neurobasal, B27, Lipofectamine 2000, donkey anti-rabbit IgG-Alexa 488, donkey anti- goat IgG-Alexa 568 conjugates were from Invitrogen. DMEM high glucose was from Mediatech. FBS was from Phoenix Research Products. HEPES, poly-L-lysine, AraC and bisbenzimide H (Hoechst 33258) were from SigmaAldrich.

C15 antibody used to detect full length APP and APP C-terminal fragments was a gift from Dr. Edie Koo (UCSD). Tau PHF-1 (ser396/404) antibody was a gift from Peter Davis (Albert Einstein University). Rabbit anti-Tau $\left(\mathrm{Ser}^{396}\right.$ ) polyclonal Antibody from GenScript (Cat\# A01387). Mouse anti-GFP IgGs (sc-9996), rabbit anti-Rab5B IgGs (sc-598), mouse anti-Rab7 IgGs(sc-10, $767)$ and mouse anti-Rab11 IgGs (sc-166,912) were from Santa Cruz Biotechnology Inc. Rabbit anti-RIN3 IgGs (ab64838) were from Abcam. Rabbit anti-BIN1 IgGs (PA5-28391) and Rabbit anti-CD2AP IgGs (PA5-34687) were from ThermoFisher. Rabbit-anti-Flag IgGs (393107) were from ABR Affinity Bioreagents Inc. Mouse anti-MAP2 IgGs (MAB378) were from Millipore. Mouse anti-GAPDH IgGs (GT239) were from GeneTex. Goat anti-rabbit or anti-mouse IgG-HRP were from Jackson ImmunoResearch Laboratories Inc.

\section{Plasmids and siRNAs}

The APP-mCherry and Rab5 ${ }^{\mathrm{S} 34 \mathrm{~N}}$-mCherry expression vectors were described before [21]. The full-length cDNA of RIN3 was generated by PCR using a human peripheral blood mononuclear cells (PBMCs) cDNA library. Neuronal isoform cDNA of BIN1 and full-length of CD2AP were synthesized by Biosune Inc. All cDNA was cloned into either pEGFP-N1 or pcDNA3.4-flag vector. RIN3$\Delta \mathrm{SH} 2(\Delta 63-158$ aa), RIN3- $\Delta \mathrm{RH}(\Delta 587-732 \mathrm{aa})$, RIN3$\Delta \operatorname{Vps} 9(\Delta 703-846 \mathrm{aa}), \quad \operatorname{RIN} 3-\Delta \operatorname{Ras}(\Delta 877-963 \mathrm{aa}) \quad$ were generated from full-length RIN3 and cloned into pEGFP-N1 vector using standard molecular cloning techniques. BACE1-mCherry construct is a gift from Dr. Uptal Das (UCSD). All expression vectors were verified by sequencing.

\section{Primary neuron culture and transfection}

Basal forebrain cholinergic neurons (BFCNs) were cultured from WT and APP/PS1 transgenic mouse E18 embryos as described previously [21, 31]. Briefly, basal forebrains were dissected, dissociated, and resuspended in Neurobasal with 10\% FBS, B27, GlutaMAX, and 50 $\mathrm{ng} / \mathrm{ml} \mathrm{NGF}$ and plated for $4 \mathrm{~h}$. Maintenance medium (Neurobasal with B27, GlutaMAX, and $50 \mathrm{ng} / \mathrm{ml}$ NGF) was added to the cell culture and incubated for $24 \mathrm{~h}$ before being replaced with antimitotic medium (maintenance medium plus $1 \mu \mathrm{M} \mathrm{AraC)}$ for $12 \mathrm{~h}$, followed by switching to maintenance medium. For transfection, $0.5 \mu \mathrm{g}$ DNA and Lipofectamine 2000 were used. An $10 \%$ transfection efficiency was achieved. Cells were imaged 24-48 h after transfection. 


\section{Cell culture and transfection}

HEK293T cells or PC12M cells were cultured in DMEM high glucose with 10\% FBS [32]. Cells were plated into 12-well/6-well culture plates $24 \mathrm{~h}$, and the confluency of the cells were about $80 \%$ prior to transfection. For plasmid transfection, Lipofectamine 2000 were used according to the manufacture's protocol. siRNAs were transfected with Lipofectamine iRNAmax. An approximately $60-70 \%$ transfection efficiency was achieved. Cells were harvested 24-48 h after transfection.

\section{Immunofluorescence}

BFCNs or PC12M cells cultured on PLL-coated glass coverslips were fixed in $4 \%$ PFA for $15 \mathrm{~min}$ at $37^{\circ} \mathrm{C}$ and were permeabilized and blocked with $0.15 \%$ Triton X100 in 3\% donkey serum in PBS for 15 min. Cells were incubated with primary antibodies (1:100 for Rab5, Rab7, Rab11; 1:200 for Flag, 1:50 for BIN1 and 1:50 for CD2AP, $1 / 200$ for MAP2) overnight at $4{ }^{\circ} \mathrm{C}$. Cells were rinsed and incubated with secondary antibody for $1 \mathrm{~h}$ at room temperature. Nuclei were stained with Hoechst $33258(0.1 \mu \mathrm{g} / \mathrm{ml})$ for $10 \mathrm{~min}$. All images were collected with a Confocal Laser Scanning Microscope (Leica).

\section{Co-immunoprecipitation and mass spectrum}

HEK293T cells cultured in $6 \mathrm{~cm}$ dish were transfected with RIN3-Flag vector and were harvested $48 \mathrm{~h}$ post transfection. Cells were solubilized with lysis buffer (150 $\mathrm{mM} \mathrm{NaCl}, 50 \mathrm{mM}$ Tris- $\mathrm{HCl} \mathrm{pH}=7.5,1 \%$ (w/v) Triton and $1 \mathrm{mM}$ phenylmethanesulfonyl fluoride). Protein lysates were extracted and incubated with antiDYKDDDDK G1 affinity Resin (GenScript, L00432) and incubated at $4{ }^{\circ} \mathrm{C}$ for $30 \mathrm{~min}$. The samples were centrifuged at $1000 \mathrm{rpm}$ for $1 \mathrm{~min}$ and the supernatant was discarded. The resin was washed three times with washing buffer containing $150 \mathrm{mM} \mathrm{NaCl}, 50 \mathrm{mM}$ Tris- $\mathrm{HCl}$ $(\mathrm{pH}=7.5)$. Proteins were eluted from the resin with DYKDDDK peptide. Mass spectrometry was performed to analyze compositions of the RIN3-containing protein complexes.

\section{Co-immunoprecipitation and Western blotting}

RIN3-GFP was co-transfected with either BIN1-Flag or CD2AP-Flag into HEK293T cells cultured in $6 \mathrm{~cm}$ dishes. The cells were harvested after $24 \mathrm{~h}$ post transfection, washed twice with PBS and solubilized with $500 \mu \mathrm{l}$ of lysis buffer as described above. Protein lysates were extracted and incubated with either anti-Flag IgGs $(0.5 \mu \mathrm{g})$ or control IgG $(0.5 \mu \mathrm{g})$ overnight at $4{ }^{\circ} \mathrm{C} .40 \mu \mathrm{l}$ protein $\mathrm{A} / \mathrm{G}$ agarose beads were added and incubated for $2 \mathrm{~h}$ at $4{ }^{\circ} \mathrm{C}$. Samples were centrifuged at $1000 \mathrm{rpm}$ centrifuge and supernatant was discarded. The resin was washed three times with washing buffer. Proteins were eluted from the resin with $40 \mu \mathrm{l}$ SDS loading buffer and boiled at $95^{\circ} \mathrm{C}$ for $5 \mathrm{~min}$. Immunoblotting was performed with anti-GFP antibody and Anti-Flag antibody.

\section{Yeast two-hybrid assay}

Yeast two-hybrid assays were performed as previously described [33]. Colonies containing the L40 strain harboring pBTM116 and PACT2 (Clontech) fusion plasmids were selected on Leu-Trp plates. $\beta$-Galactosidase activities were measured by a liquid assay.

\section{Live cell imaging, axonal transport of APP and BACE1}

APP-mCherry, BACE1-mCherry or mCherry was transfected into mouse E18 primary cortical neurons at DIV4-5. For co-transfection, EGFP, RIN3-GFP, BIN1GFP and CD2AP-GFP constructs were co-transfected with mCherry or APP-mCherry or BACE1-mCherry and incubated overnight. Time-lapse images were taken at 1 frame $/ 3 \mathrm{~s}$ for a total of $90 \mathrm{~s}$. $24 \mathrm{~h}$ post transfection, timelapse images were captured at 1 frame/sec for a total of 2 min per series using a Leica DMI6000B inverted microscope, which is equipped with an environmental chamber $\left(37^{\circ} \mathrm{C}\right)$ as described previously [21, 34]. Both fluorescent images and DIC images were also captured for analysis. Generation of kymographs and analysis of transport were performed using ImageJ [21, 34].

\section{Statistical analysis}

All experiments were repeated at least 3 times independently. Data represent mean \pm SEM. Statistical analyses and calculation of $p$ values were performed using Prism6(GraphPad Software, La Jolla, CA); Standard $t$ test was used for pairwise comparisons and two-way ANOVA for multiple comparisons. $p$ values less than 0.05 were considered statistically significant, and $p$ values less than 0.01 were considered statistically highly significant.

\section{Results \\ APP/PS1 mice show increased RIN3 expression and early endosome enlargement}

Given that GWAS analysis and methylation profile study revealed that expression of RIN3 is likely upregulated in $\mathrm{AD}[13,24]$, we decided to investigate if this was also true in a mouse model of $\mathrm{AD}$. We elected to measure both RIN3 mRNA and protein level in the APP/PS1 mouse model of AD. The APP/PS1 model expresses a chimeric mouse/human amyloid precursor protein $(\mathrm{Mo} /$ HuAPP695swe) and a mutant human presenilin 1 (PS1dE9). Both APP695swe and PS1-dE9 mutations are associated with early-onset Alzheimer's disease [35-37].

We first used quantitative PCR (qPCR) to measure the mRNA level in different brain region of APP/PS1 mice from 1 week old to 9 months of age and compared the measurements with their age-gender matched wild type (WT) mice. As shown in Fig. 1a, APP/PS1 mice showed 


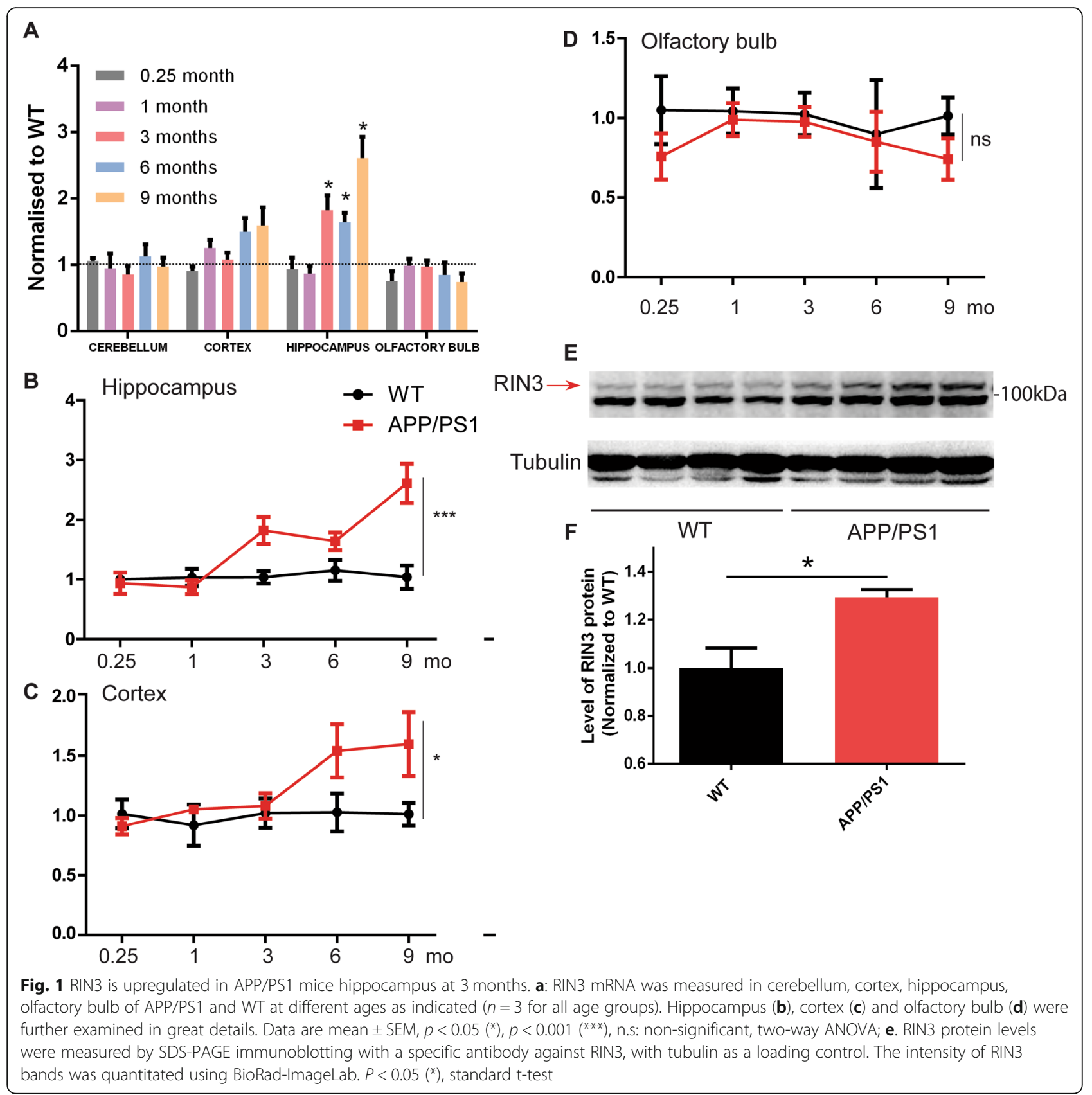

no significant changes in RIN3 mRNA level in either the cerebellum or the olfactory bulb (Fig. 1a, d). However, RIN3 mRNA was significantly increased in both the cortex and the hippocampus (Fig. 1a-c), two brain regions that are markedly impacted in AD. The increase in RIN3 mRNA in the hippocampus became significantly evident as early as at 3 months of age (Fig. 1b). Similarly, starting at 3 months of age, RIN3 mRNA level in the cortex increased and reached a significant difference at 6 months of age (Fig. 1c). These results are significant since these mice do not develop $\beta$-amyloid deposits in their brain until they reach 6 to 7 months of age. We thus conclude that upregulation of RIN3 precedes $\beta$-amyloid deposits in the APP/PS1 AD mice.

To define if expression of RIN3 at the protein level was also increased in APP/PS1 mice, we used immunoblotting to measure RIN3 protein in the hippocampus of 3 months old male APP/PS1 mice. Our results confirmed that the RIN3 protein level (Fig. 1e, f) showed an increase in these mice as compared to age-matched WT littermates. Taken together, our results have demonstrated that expression of RIN3 is increased in early development of APP/PS1 mice, well preceding the appearance of $A \beta$ plaques in these mice. 
BFCNs are believed to have some of the longest axons [38-44]. These large axonal projections make BFCN neurons extremely vulnerable, it is thus not surprising that basal forebrains degenerate early in AD [39]. Previously, we showed that Rab5 endosomes were enlarged in BFCNs when APP was overexpressed [21]. We cultured
E18 BFCNs from APP/PS1 and their WT control embryos. We first stained for Rab5 using a specific antibody. Our results showed that the size of Rab5 vesicles were indeed increased in APP/PS1 neurons as compared to WT neurons (Fig. 2b versus a). This became very evident by the quantitation of both the diameters (Fig. 2c)
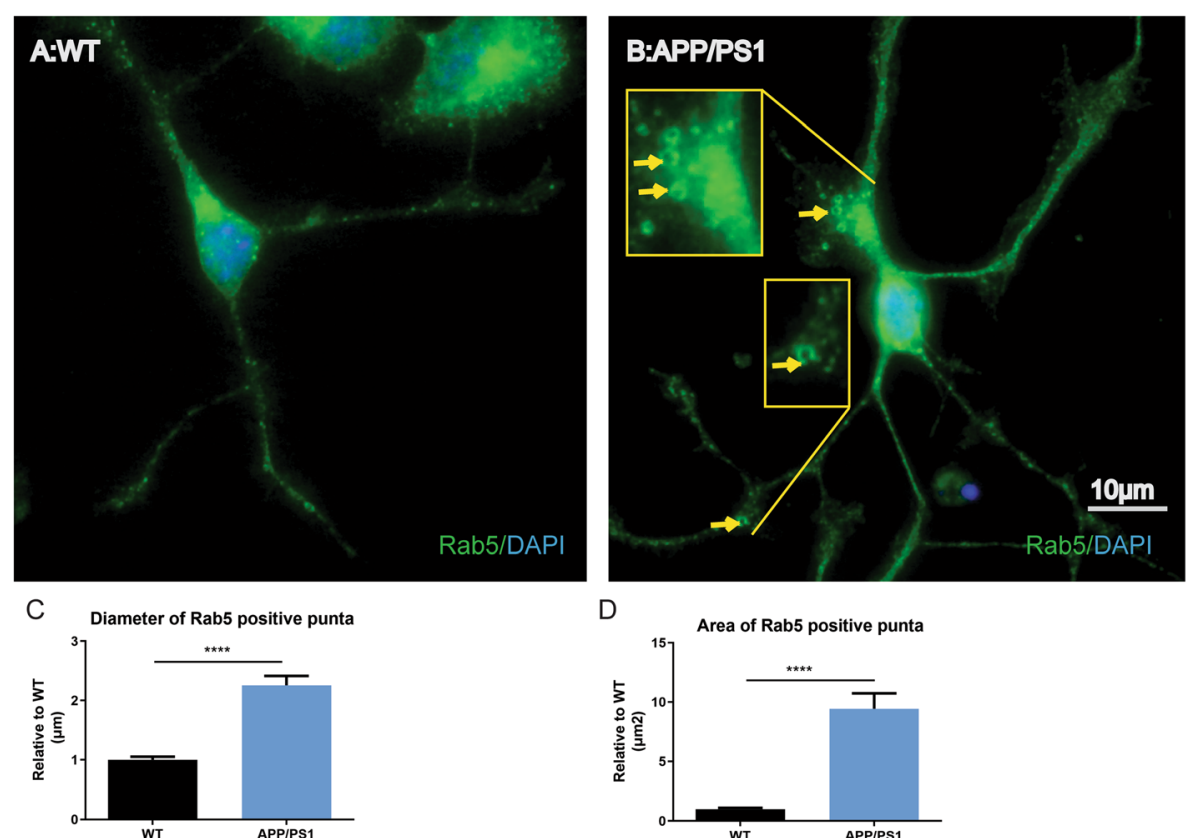

D Area of Rab5 positive punta
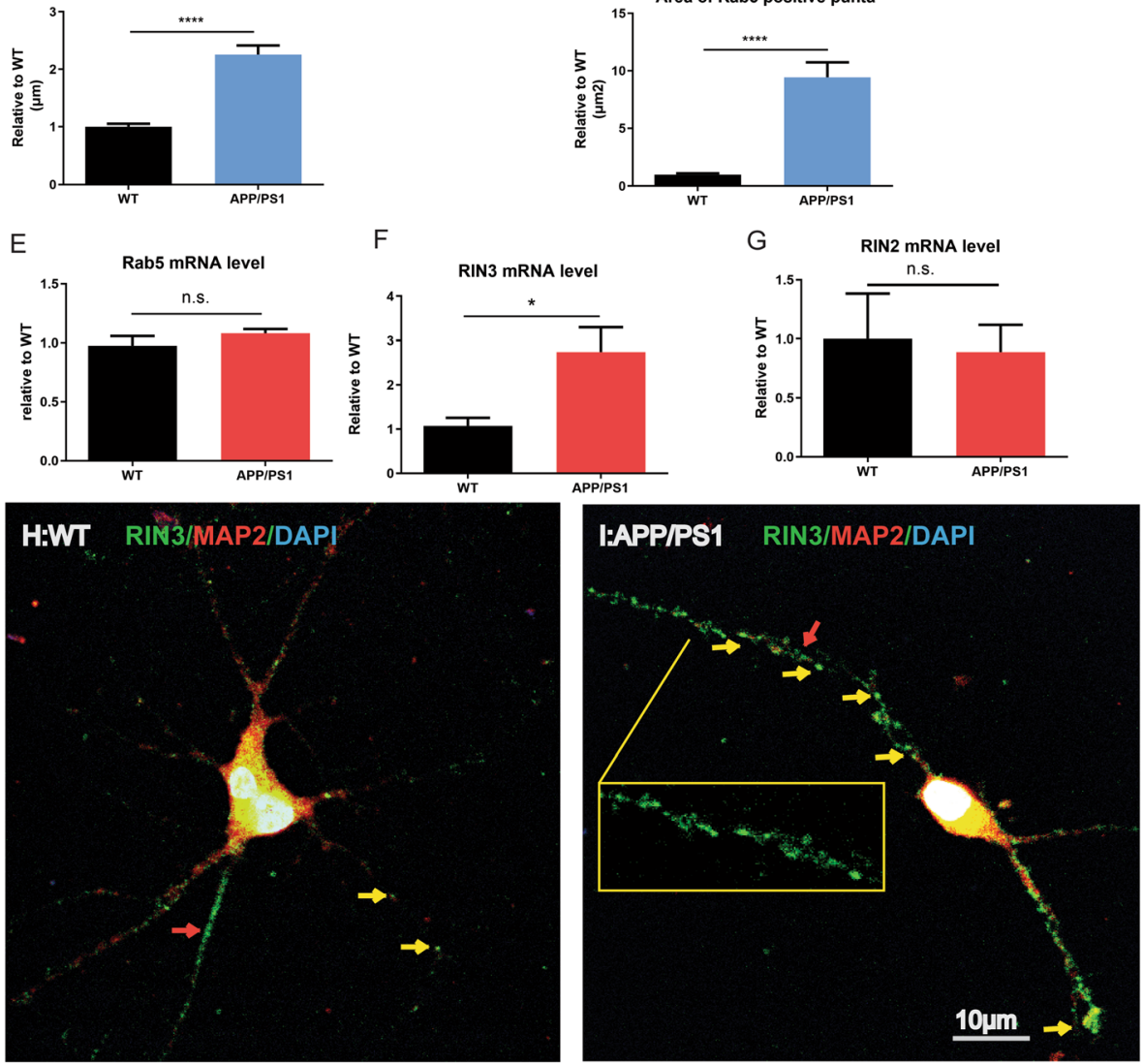

Fig. 2 RIN3 upregulation in APP/PS1 BFCNs induce enlarged early endosome. E18 BFCNs of WT (a) and APP/PS1 (b) were cultured and fixed at DIV4 for immunostaining with Rab5 antibody. Nuclei were stained with DAPI. Rab5 positive vesicles were measure by diameter (c) and size (d) using ImageJ (in blue bar graph). The mRNA level for Rab5 (e), RIN3 (f) and RIN2 (g) were quantitated using qPCR (in red bar graph). Two tail Ttest was used. ${ }^{*} \mathrm{p}<0.05$ and ${ }^{* *} p<0.001$. As in A and B, E18 BFCNs of WT (h) and APP/PS1 (i) were also co-stained for RIN3 and MAP2. The inset in I shows enlarged RIN3 puncta. Representative images are shown 
and areas of Rab5 endosomes (Fig. 2d). However, qPCR analysis showed no increased in Rab5 at the message RNA level (Fig. 2e).

To define if RIN3 expression was also increased in BFCN neurons that tracked early endosomal pathologies in cultured APP/PS1 BFCNs, we also measured the RIN3 mRNA level by qPCR. Intriguingly, APP/PS1neurons showed a significant increase in RIN3 expression (Fig. 2f). To determine if the increase was specific for RIN3, we also measured the expression level of RIN2(Fig. 2h), another member of the RIN family. Our results showed no changes in the RIN2 mRNA level. Therefore, RIN3 is selectively upregulated in cultured BFCN neurons of APP/PS1 mice. Since RIN3 is a GEF for Rab5, increased RIN3 expression likely contributes to enlarged Rab5 early endosomes in cultured BFCNs from APP/ PS1.

To further define the subcellular localization of RIN3Rab5 vesicles, we co-stained BFCN for RIN3 [21] and MAP2, a somal/dendritic marker using specific antibodies. As shown in Fig. 2h, i, RIN3 positive puncta were present in soma and dendrites (both are immunoreactive to anti-MAP2 antibody) (Fig. 2h, i). RIN3 puncta were also seen in processes that were negative for MAP2. i.e. axons (indicated by arrows) (Fig. 2h). Further, the size of RIN3 puncta in APP/PS1 BFCNs appeared to be enlarged in comparison to WT neurons (Fig. $2 \mathrm{i}$ versus $\mathrm{h}$, also see the insert). Together, our results have revealed that a selective increase in RIN3 expression may induce enlargement of Rab5-early endosomes in cultured E18 mouse BFCNs of APP/PS1 mice.

\section{RIN3 interacts with two other AD risk factors, CD2AP and BIN1}

To further understand the biological function of RIN3, we expressed a flag-tagged RIN3 construct in HEK293T cells. RIN3-containing protein complexes were purified and analyzed by Mass Spectrometry to identify the RIN3 interactome. A total of 380 proteins were positively identified to interact with RIN3. Followed by a GO analysis, the RIN3 interactome was divided into 20 groups (Fig. 3a). The RIN3-interactome is highly enriched of proteins (Peptide Spectrum Match, PSM value 25 ) that mediate vesicle transport. This is illustrated by a protein interaction network (Fig. 3b).

As expected, many of these RIN3-interacting proteins have been implicated to regulate endocytosis and intracellular vesicular trafficking. Interestingly, our analysis has uncovered that two other AD risk factors were highly associated with RIN3; BIN1 (bridging integrator 1) $(\mathrm{PSMs}=$ 22 , coverage $=38 \%$ and detected peptides $=15)$ and CD2AP $(\mathrm{CD} 2$-associated protein $)(\mathrm{PSMs}=21$, coverage $=$ $30 \%$ and detected peptides $=17)($ Supplementary Table 1$)$. Thus, RIN3 likely forms a complex with BIN1/CD2AP.
To confirm the interaction, we conducted coimmunoprecipitation (IP) assay. We co-transfected RIN3-GFP with either CD2AP-flag or BIN1-flag into HEK293T cells. An anti-flag antibody was used to immunoprecipitated CD2AP or BIN1. To ensure the pulldown efficiency of the anti-flag antibody, we assayed the cell lysate inputs, the post-IP supernatant and the IP products. As shown in Fig. 3c, protein lysates from cells transfected with the BIN1-flag construct, but not from the un-transfected cells, gave rise to the expected BIN1flag protein as recognized by the anti-flag antibody by immunoblotting (Fig. 3c, top panel). In the post-IP supernatant, the BIN1-flag product was still detected in samples that incubated with a control $\operatorname{IgG}(\operatorname{IgG})$, but the same band was no longer seen in samples incubated with anti-flag (Fig. 3c, middle panel). As expected, BIN1flg was only present in the pulldowns with anti-flag but not with the control IgG (Fig. 3c, bottom panel). These data have demonstrated a great efficiency of the anti-flag antibody in IP assays. We then performed the co-IP experiments. Our analysis of the IP complexes has showed that RIN3-GFP was co-immunoprecipitated when coexpressed with either CD2AP-flag (Fig. 3d) or with BIN1-flag (Fig. 3e). Our data are evidence that BIN1 and CD2AP both interact with RIN3. The interaction between RIN3 and BIN1/CD2AP was further confirmed in yeast two-hybrid assays (Fig. 3f). Significantly, no interaction between BIN1 and CD2AP was detected in these assays (Fig. 3f). These results have thus demonstrated that RIN3 directly interacts with BIN1 and CD2AP either simultaneously or separately.

To further corroborate our IP data that RIN3 interacted with BIN1 and CD2AP, we transfected RIN3-GFP in PC12 cells (Fig. 4a, b) and mouse E18 embryonic primary neurons (Fig. 4c, d). We then used specific antibodies against BIN1 or CD2AP to detect the subcellular distribution of endogenous BIN1 and CD2AP with respect to RIN3-GFP by immunostaining. Of note, we consistently observed enlarged vesicles following expression of RIN3-GFP. Furthermore, both endogenous BIN1 and CD2AP were recruited to these vesicles and colocalized with RIN3(Fig. 4a, b). In primary cultured neurons, RIN3-BIN1 and RIN3-CD2AP puncta were mostly seen in processes (axons and dendrites), but not in cell bodies (Fig. 4c, d). Consistent with previous reports [26, 45, 46], these findings suggest that the RIN3-BIN1-CD2AP complex is likely involved in mediating intracellular vesicular sorting and trafficking.

Structurally, RIN3 has a Src homology 2 (SH2) domain, a proline rich domain (PRD), a RIN-homology (RH) domain, a Vps9 (vacuolar protein sortingassociated protein 9) and a Ras-association (RA) domain $[26,46]$. Previous studies have shown that BIN1 and CD2AP interacted with RIN3 through their SH3 domain 
A

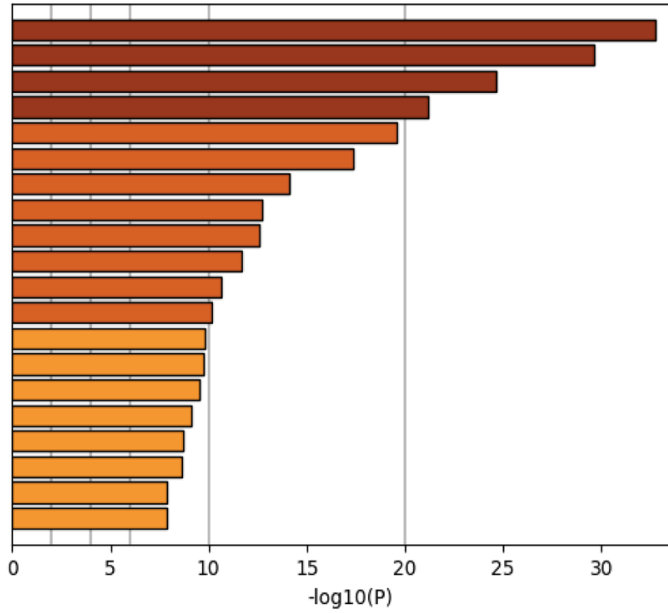

GO:0072594: establishment of protein localization to organelle R-HSA-2262752: Cellular responses to stress

GO:0006457: protein folding

R-HSA-1640170: Cell Cycle

R-HSA-3371497: HSP90 chaperone cycle for steroid hormone receptors (SHR)

GO:0044403: symbiont process

R-HSA-5653656: Vesicle-mediated transport

GO:0050821: protein stabilization

M186: PID PDGFRB PATHWAY

GO:0006986: response to unfolded protein

GO:1903827: regulation of cellular protein localization

R-HSA-449147: Signaling by Interleukins

R-HSA-6798695: Neutrophil degranulation

M66: PID MYC ACTIV PATHWAY

R-HSA-9006934: Signaling by Receptor Tyrosine Kinases

GO:0006839: mitochondrial transport

GO:0044257: cellular protein catabolic process

R-HSA-187037: Signaling by NTRK1 (TRKA)

GO:0031329: regulation of cellular catabolic process

GO:0000302: response to reactive oxygen species

B

Vesicle-mediated transport related proteins interact with RIN3

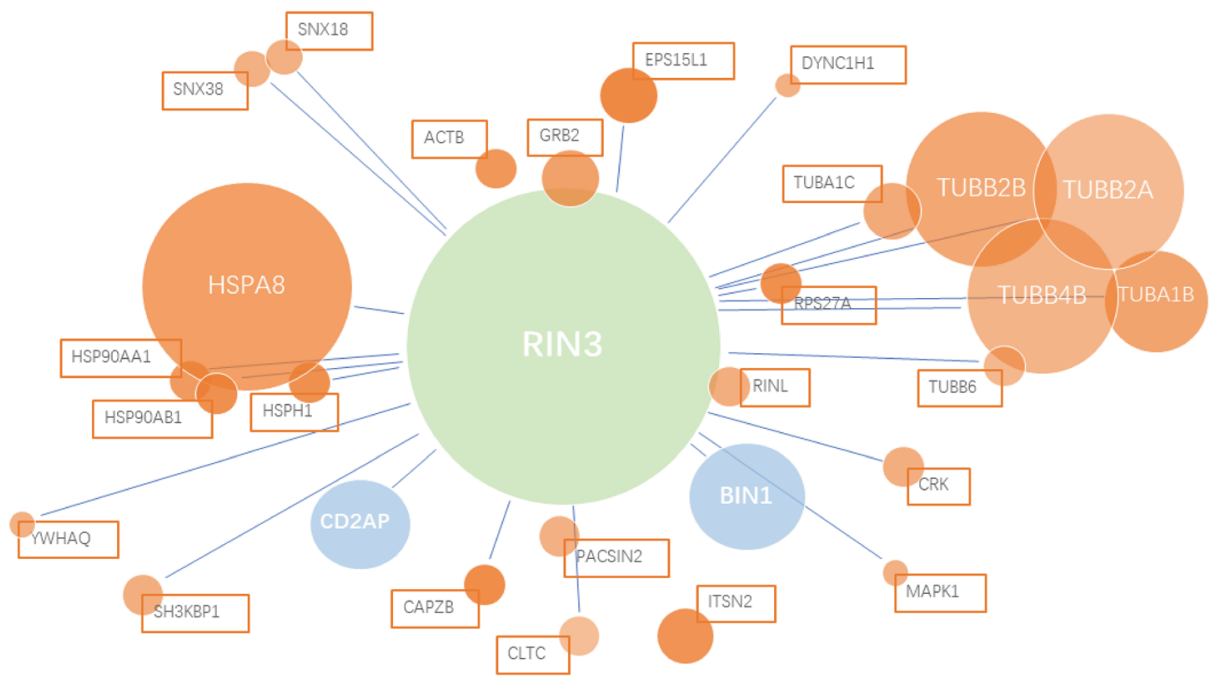

C

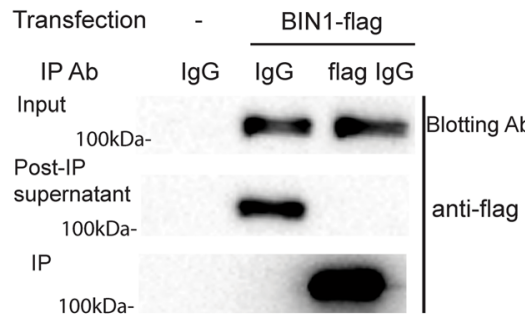

D

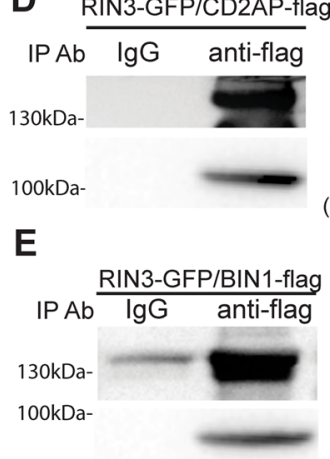

$\mathbf{F}$

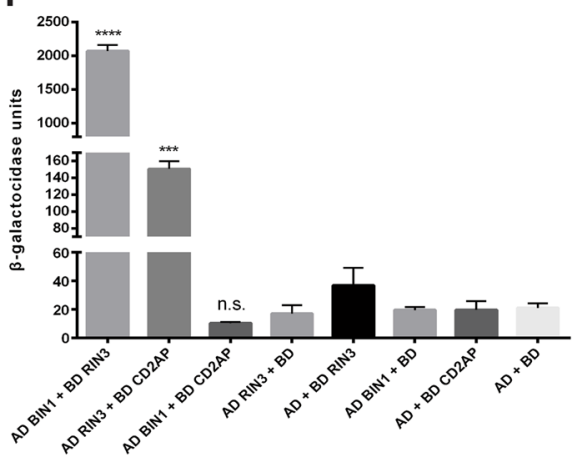

Fig. 3 (See legend on next page.) 
(See figure on previous page.)

Fig. 3 BIN1 and CD2AP interact with RIN3. RIN3-flag was expressed and purified from HEK293t cells, then MS was used to identify and define RIN3 interactomes. a: A set of 380 RIN3-interacting proteins were detected, and a GO analysis was conducted. The RIN3 interactomes were divided into 20 categories with respect to different cellular function. b: A protein interaction network highlighting the RIN3-interactome that regulate endocytic/vesicular transport, based on 28 highly enriched proteins (PSM value $\geq 5$ ). The circle size indicated the degree of enrichment. RIN3 was in green, LOAD risk factors were in blue. Interaction between RIN3 and CD2AP/BIN was analyzed by co-IP. HEK293 cells were transfected with indicated vectors. Cell lysates were incubated with either control lgG (IgG) and anti-flag IgG. Untransfected cells were used as a control. In $\mathbf{c}$, the cell lysate inputs, the post-IP supernatant and the IP complexes were analyzed by SDS-PAGE/immunoblotting with indicated antibody. In d, RIN3-GFP was co-IP with CD2AP-flag; In e, RIN3-GFP was co-IP with BIN1-flag. For both D, E, a control lgG (IgG) was used as a negative control. RIN3-GFP was blotted with a GFP antibody, an anti-flag antibody was used to detect CD2AP-flag (d) or BIN1-flag (e). e Yeast two hybrid assay was performed, each group had three replicants. Standard t-test, ${ }^{* * *}$ stands for $p<0.0001$, *** stands for $p<0.001$, n.s. stands for $p>0.05$

[26, 47]. We performed further immunofluorescence to define the domain(s) of RIN3 was responsible for its interaction with BIN1 and CD2AP. We selectively deleted each of the following domains: $\mathrm{SH} 2, \mathrm{RH}, \mathrm{Vps} 9$, or Ras from RIN3 and constructed RIN3- $\Delta$ SH2-GFP, RIN3- $\Delta$ RH-GFP, RIN3- $\Delta$ Vps-GFP, RIN3- $\Delta$ Ras-GFP vectors, respectively (Figure S1). We then transfected each of these constructs with BIN1-flag or CD2AP-flag into PC12M cell line. Our data showed that deletion of each of these four domains had no effect on the interaction between RIN3 and BIN1 or between RIN3 and CD2AP, since BIN1-flag, CD2AP-flag or Rab5 were still colocalized with RIN3- $\triangle$ SH2-GFP, RIN3- $\triangle$ RH-GFP, RIN3$\Delta$ Vps-GFP and RIN3- $\Delta$ Ras-GFP (Figure S1). However, deletion of the $\mathrm{SH} 2$ or the $\mathrm{RH}$ domain did result in diffused localization for RIN3, BIN1, CD2AP and Rab5 throughout the cytoplasm. These results suggest that the $\mathrm{SH} 2$ or the $\mathrm{RH}$ domain is required for targeting of RIN3 along with BIN1, CD2AP to Rab5 endocytic vesicles. We therefore conclude that either the N-terminal $\mathrm{SH} 2$ domain or the PRD domain of RIN3, or both, are likely required for mediating the interaction between RIN3 and BIN1 and CD2AP and target these two proteins to early endocytic vesicles. Taken together with our biochemical, genetic and cellular studies, our results suggest that the three AD risk factors: RIN3, BIN1 and CD2AP may form a functional complex to impact endocytic sorting and trafficking.

\section{Upregulation of RIN3 induces recruitment of CD2AP and BIN1 to early endosomes}

We next investigated the potential impact(s) of increased expression of RIN3 on BIN1 and CD2AP with respect to Rab5. BIN1 has been shown to be involved in the formation of recycling endosomes from early endosomes [11]. CD2AP is an adaptor that stabilizes internalized receptors in early endosomes, as such, either to prevent receptors from being degraded or to facilitate the transmission of certain signals [45, 48]. Based on our findings that RIN3 interacted with both CD2AP and BIN1, RIN3 was localized to early endosomes, we thus postulated that RIN3 recruited CD2AP and BIN1 to early endosomes. To investigate this, we examined the cellular localization of CD2AP, BIN1 with or without co-expression of RIN3. In the absence of RIN3 expression, the signals for CD2AP-flag (Fig. 5a, b) and BIN1flag (Fig. 5e, f), as revealed by IF, were mostly diffused across the cytoplasm in PC12 cells, with little or no presence on Rab5-early endosomes (Fig. 5a, e). By cotransfection with RIN3-GFP, the signals for CD2AP (Fig. 5c, d) and BIN1 (Fig. 5g, h) became colocalized with Rab5, but not with Rab7 (Figure S2) or Rab11 (Figure S3). Of note, colocalization of the three different colors is indicated by the appearance of peaks of fluorescence intensities at the same position(s), as shown in Fig. 5d and $\mathrm{h}$ (red arrow heads). Likewise, non-colocalization is revealed by the three peaks that are randomly distributed as in Fig. $5 \mathrm{~b}$ and $\mathrm{f}$. These results provide further evidence that increased expression of RIN3 recruits CD2AP and BIN1 specifically to early endosomes, but not to late endosomes or recycling endosomes.

\section{Role of RIN3, BIN1 and CD2AP in APP and BACE1 trafficking}

RIN3, BIN1 and CD2AP have all been implicated in regulating endocytic trafficking. To define their role in axonal transport, we expressed APP-mCherry or BACE1mCherry in mouse E18 primary cortical neurons either alone or with RIN3-GFP, BIN1-GFP and CD2AP-GFP, respectively. As a control, we first examined APP-mCherryor BACE1-mCherry in axons of neurons co-transfected with EGFP. The signals for EGFP were diffused while the signals for APP-mCherry or BACE1-mCherry appeared as red puncta (Fig. 6, a, e, also Figure S4A). Similar results were found in axons of neurons co-transfected with BIN1GFP and APP-mCherry or BACE1-mCherry (Figure S4A, $B)$. In contrast, neurons that express either RIN3-GFP or CD2AP-GFP showed distinct features in their axons from those of expressing BIN1-GFP. The signals for RIN3-GFP and CD2AP-GFP were overlapped significantly with either APP or BACE1 puncta (Figure S4B, C).

To ask whether or not overexpression of RIN3, BIN1 or CD2AP impacted axonal transport, we examined axonal transport of APP-mCherry (Fig. 6, a, c, e, g) and 


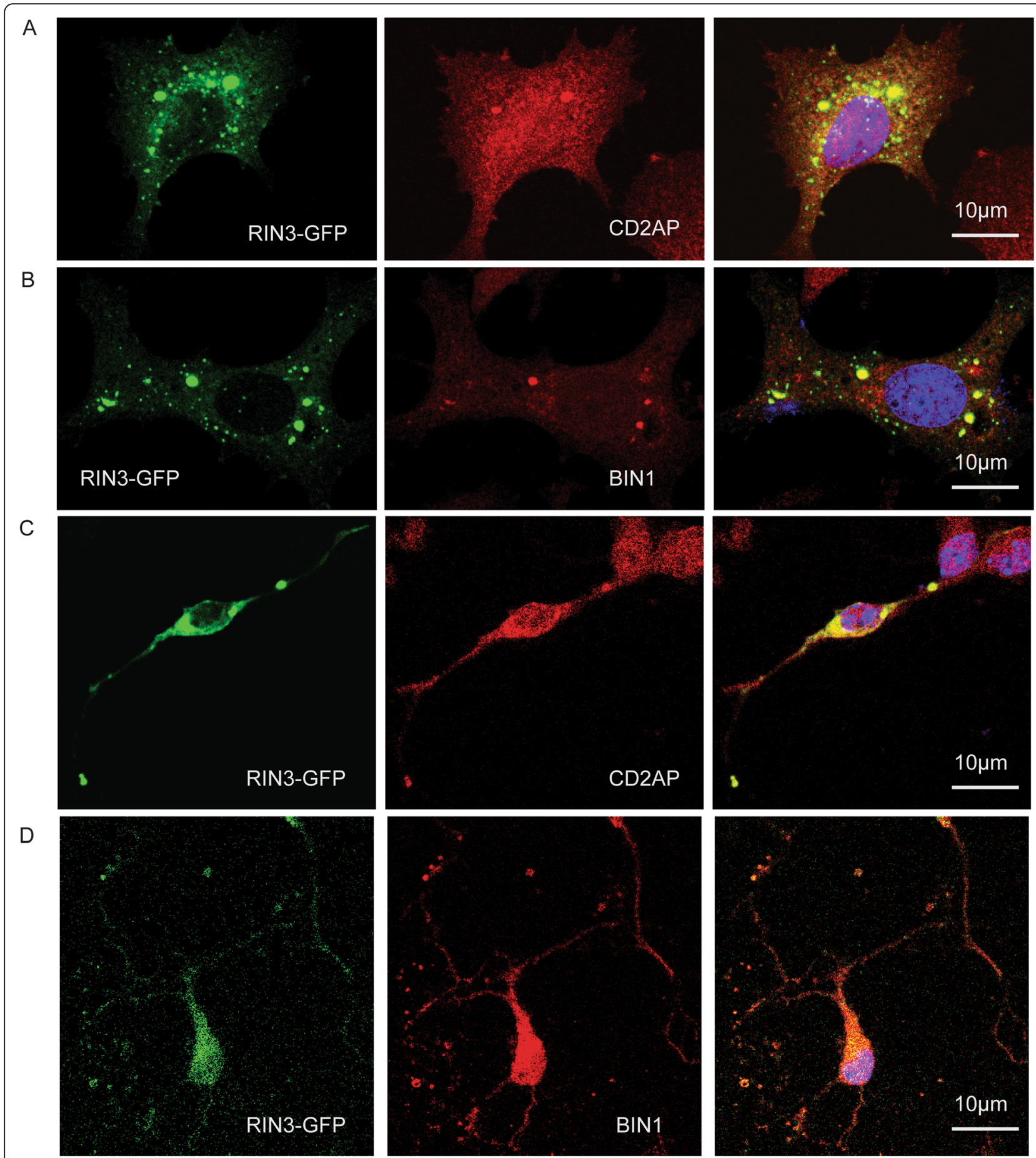

Fig. 4 RIN3 colocalizes with CD2AP and BIN1. PC12M cells (a and b) and primary cortical neurons (c and $\mathbf{d}$ ) were transfected with RIN3-GFP, followed by immunostaining for CD2AP (a, $\mathbf{c})$ or BIN1 $(\mathbf{b}, \mathbf{d})$ or CD2AP using specific antibodies. Yellow color denotes colocalization. Representative images are shown

BACE1-mCherry (Fig. 6, b, d, f, h) by live imaging $24 \mathrm{~h}$ after transfection. Time-lapse image series were captured, and kymographs were generated; representative images with corresponding kymographs for each condition are shown (Fig. 6). When compared with EGFP transfected neurons, cortical neurons expressing either RIN3-GFP or CD2AP-GFP showed a significant reduction in both the percentile of mobile puncta and the average transport velocities for APP and BACE1 (Fig. 6 i-1). In RIN3-GFP transfected neurons, the average 

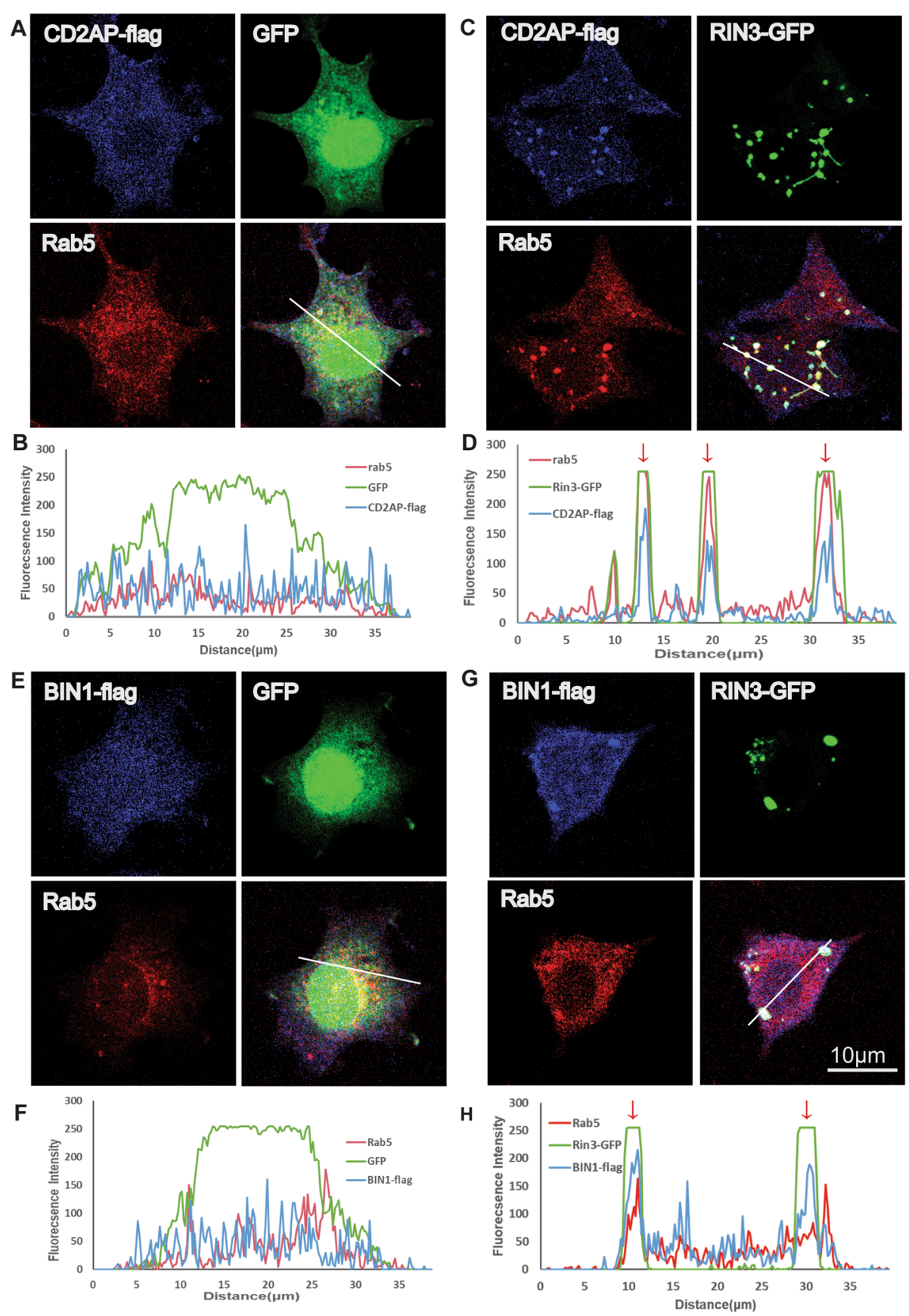

Fig. 5 CD2AP and BIN1are recruited to early endosome by RIN3. PC12M cells expressing GFP/CD2AP-flag (a, b), RIN3-GFP/CD2AP-flag (c, d), GFP/ BIN1-flag (e, f), RIN3-GFP/BIN1-flag (e-h) were immune-stained for Rab5 with a specific antibody. Representative images are shown. Colocalization analysis was performed by image by measuring fluorescence intensity alongside the drawn line. The three different colors peak at the same position If the three proteins were colocalized $(\mathbf{b}, \mathbf{d}, \mathbf{f}, \mathbf{h})$

transport velocities of APP-mCherry and BACE1mCherry particles were significantly reduced by approximately $30.2 \%(p=0.0222)$ and $55.3 \%(\mathrm{p}<0.0001)$, respectively; And the percentiles of moving puncta were also significantly reduced by $9.54 \%(p=0.0083)$ and $20.5 \%$ $(\mathrm{p}=0.0083)$, respectively (Fig. 6i-l). The average moving velocities of APP-mCherry and BACE1-mCherry particles seen in CD2AP-GFP transfected neurons were also reduced by $31.4 \%(p=0.002)$ and $52.7 \%(p<0.0001)$ while the decrease in the percentiles of mobile puncta were not significant $(p=0.2954$ and $p=0.1435$ ) (Fig. 6i1). Neither the moving velocities nor the percentile of mobile puncta for APP-mCherry and BACE1-mCherry exhibited significant difference from either the control 

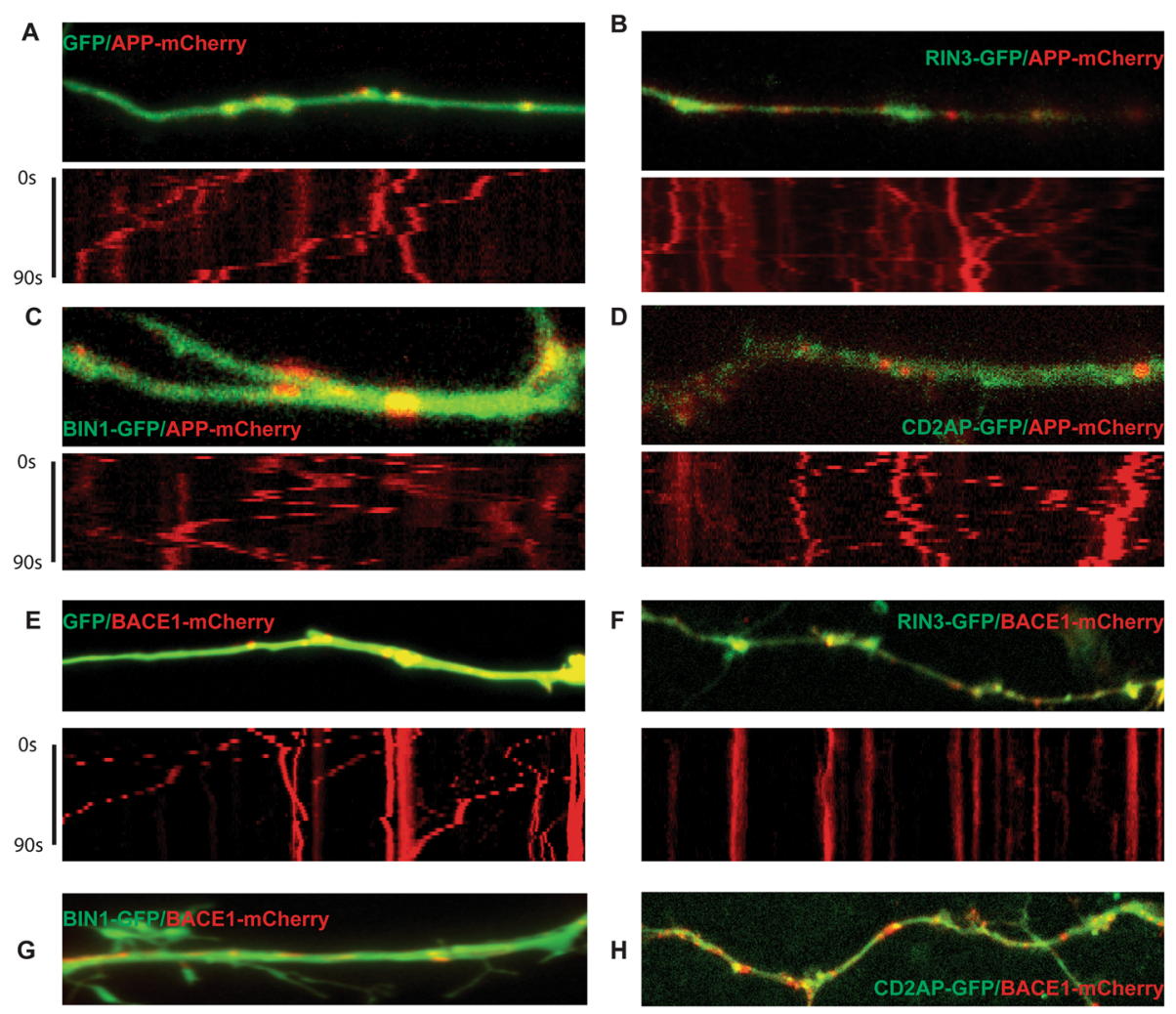

H
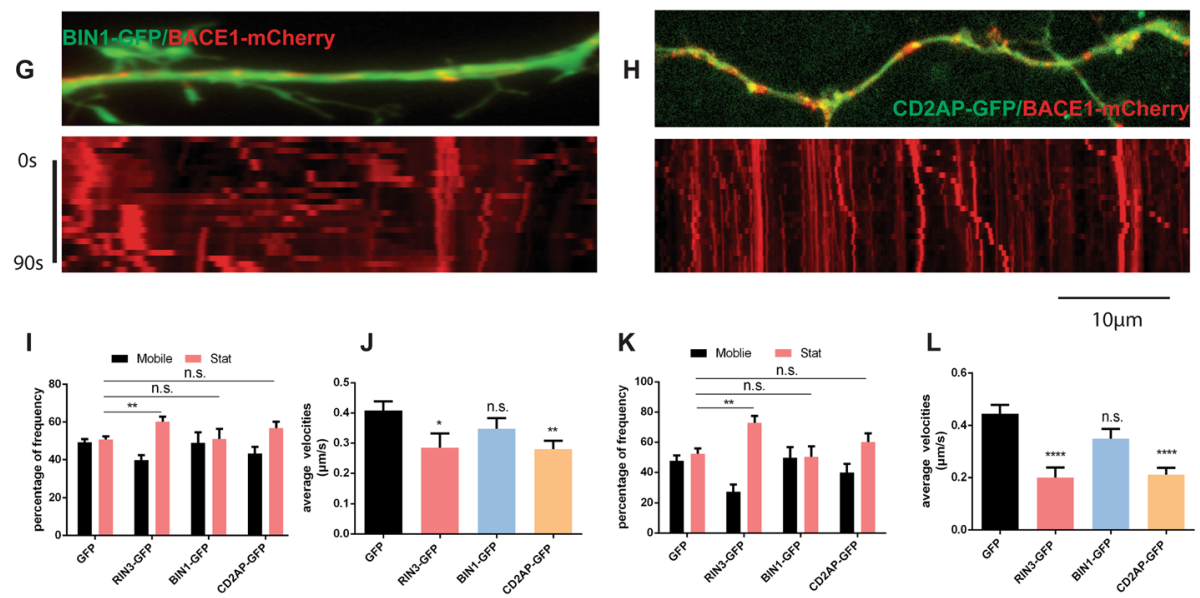

Fig. 6 RIN3 and CD2AP inhibited transport of APP and BACE1 in primary cortical neurons. E18 mouse cortical neurons were cultured in microfluidic chambers and were co-transfected with the indicated expression vectors. Live imaging was performed as described in the Methods section. Representative images of axons and corresponding kymographs are shown in $\mathbf{a}-\mathbf{h}$. The percentiles of mobile versus stationary vesicles (stat) (i, k) and average velocities ( $\mathbf{g}, \mathbf{l})$ for APP-mCherry and BACE1-mCherry in axons were quantitated. Data represent mean \pm SEM of at least 3 independent experiments. All $p$-values were calculated using 1-way ANOVA. $p<0.05\left({ }^{*}\right), p<0.01\left({ }^{* *}\right), p<0.0001(* * *)$, $p>0.05$ (n.s.), standard t-test

or from BIN1-GFP expressing neurons $(p=0.192$ and $p=0.0797)($ Fig. 6i-l).

\section{Role of RIN3/BIN1/CD2AP in APP processing and tau phosphorylation}

Our current study has suggested a strong possibility that RIN3 may function as a scaffold to tether BIN1 and CD2AP on Rab5-early endosomes to regulate axonal trafficking. Our earlier observations have shown that RIN3 expression (both mRNA and protein) was increased in both cultured E18 BFCNs and 3-month-old brain lysates in APP/PS1 mice (Figs. 1 and 2). To define if BIN1 and CD2AP expression were also altered in APP/PS1 mice $[49,50]$, we performed immunoblotting with specific antibodies against BIN1 or CD2AP using brain lysates of 3 month-old APP/PS1 mice and their age- gender matched WT mice. Interestingly, we found the neuronal variant of BIN1(Aphiphysin1) was significantly reduced with a concomitant increase in the level of non-neuronal BIN1 isoforms in the APP/PS1 brain compared to WT brain (Fig. 7a, b). For CD2AP, we saw a 2-fold increase in the APP/PS1 mice compared to WT mice $(p=0.0184)$ (Fig. 7a, b). Together, the data have demonstrated a significant alteration in the levels/ 

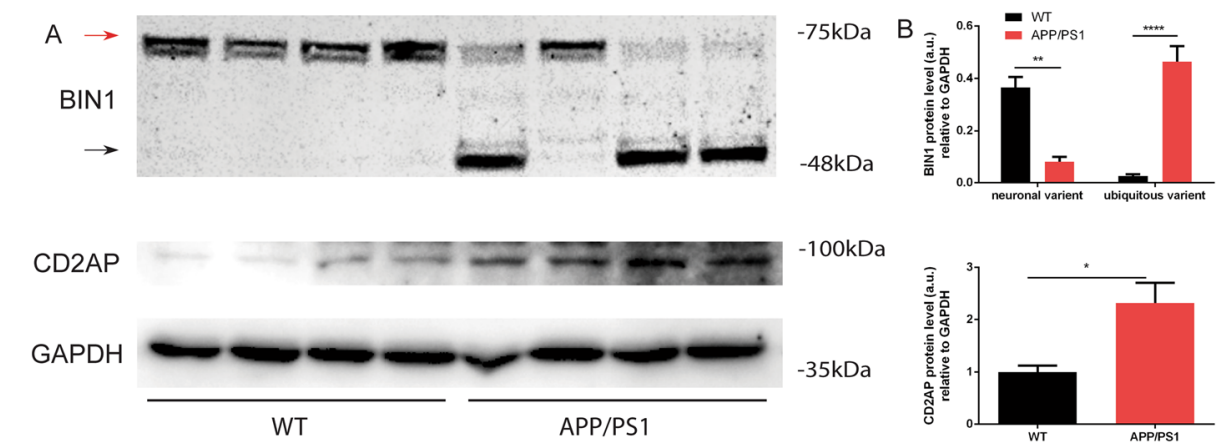

C: Cortex
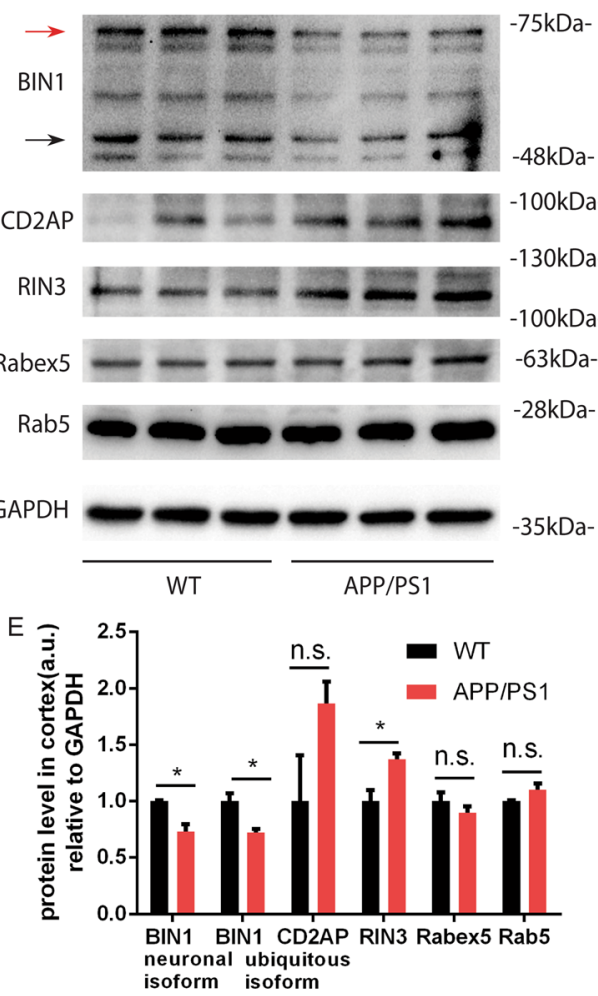

D: Hippocampus
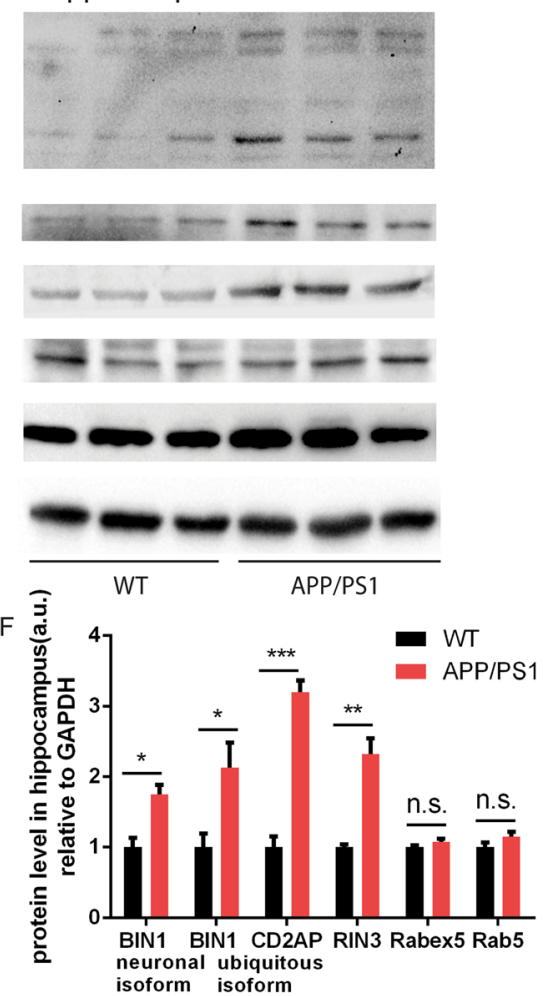

Fig. 7 BIN1 and CD2AP expression level in APP/PS1 mice. 3-month-old APP/PS1 mice and age-matched WT mice were dissected, lysates were immunoblotted for BIN1 and CD2AP (a). The protein levels were normalized against GAPDH as a loading control (b). In Cortex (c) and hippocampus (d) were also extracted from10-month-old APP/PS1 and age-matched WT mice. Protein lysates were used to probe for BIN1, CD2AP, RIN3, Rabex5 and Rab5 using indicated antibodies by SDS-PAGE/immunoblotting (c and $\mathbf{d}$ ), The intensity of bands was quantitated using BioRad-ImageLab. The respective protein levels were normalized against GAPDH as a loading control (e, f). $p<0.05\left(^{*}\right), p<0.01\left(^{* *}\right), p<0.0001$ $(* * *)$, standard t-test

activities of the RIN3/BIN1/CD2AP complexes in APP/ PS1 mouse brains: an increase in both RIN3 and CD2AP with a concomitant reduction in BIN1.

To further investigate developmental changes in the RIN3/BIN1/CD2AP complex, we dissected the cortex and the hippocampus from 10-month-old APP/PS1 mice and age-matched WT mice. The samples were analyzed by immunoblotting (Fig. 7). Intriguingly, both forms of BIN1 showed a decrease in the cortex (Fig. 7c, e) but an increase in the hippocampus (Fig. $7 \mathrm{~d}, \mathrm{f}$ ) in APP/PS1 as compared to WT. CD2AP was also significantly upregulated in the hippocampus but not in the cortex in APP/PS1 mice (Fig. 7d, f versus Fig. 7c, e). Consistently, RIN3 was increased in both regions in APP/PS1 mouse brain. We further examined the protein level of Rabex5, another potential GEF for Rab5. In contrast to RIN3, our results did not show an increase in the protein level of Rabex5 (Fig. 7c-f). We also did not see an increase in the protein level of Rab5 (Fig. 7cf). Together with our earlier results (Figs. 1 and 2), these data have provided evidence that RIN3 is consistently upregulated in APP/PS1 mice. 
Recent evidence has suggested a role of BIN1 and CD2AP in APP trafficking processing to regulate extracellular $A \beta$ deposition $[51,52]$. BIN1 has been shown to control axonal $\mathrm{A} \beta$ production: depletion of the neuronal BIN1 variant disrupted APP recycling process and increased $A \beta$ production within intraneuronal compartments [52]. On the other hand, CD2AP depletion induced APP accumulation in early endosomes that retarded APP degradation to enhance intraneuronal A $\beta$ generation as well [52].

To define the effect of RIN3, BIN1, CD2AP in APP processing and Tau phosphorylation, we expressed these constructs tagged with GFP in PC12 cells. GFP was used as a control. Cell lysates were immunoblotted with a rabbit antibody against the APP C-terminal 15 residues (C15). The C15 antibody recognizes the full length APP as well as various forms of APP C-terminal fragments (APP-CTFs) such as $\beta C T F$ and $\alpha$ CTF. Consistent with earlier studies [51], upregulating BIN1 had little effect on the level of $\beta C T F s$ production (Fig. 8a and c). Expression of either RIN3 or CD2AP yielded more $\beta$ CTFs in PC12 cells (Fig. 8a and c). We detected at least two bands for $\beta$ CTF, that likely corresponded to either C99 or C99 and its phosphorylated form, as reported by other investigators [53]. When probed the lysates for pTau with the PHF1 antibody, our results showed that expression of both BIN1 and RIN3, but not CD2AP, induced an increase in pTau (Fig. 8a, c).

To further investigate if the effect of RIN3 on the increase in $\beta C T F$ s and pTau was mediated by Rab5 activation, we transfected a dominant negative Rab5 construct $\left(\mathrm{Rab}^{\mathrm{S} 34 \mathrm{~N}}\right)$ into PC12 cells. Expression of $\mathrm{Rab5}^{\mathrm{S} 34 \mathrm{~N}}$

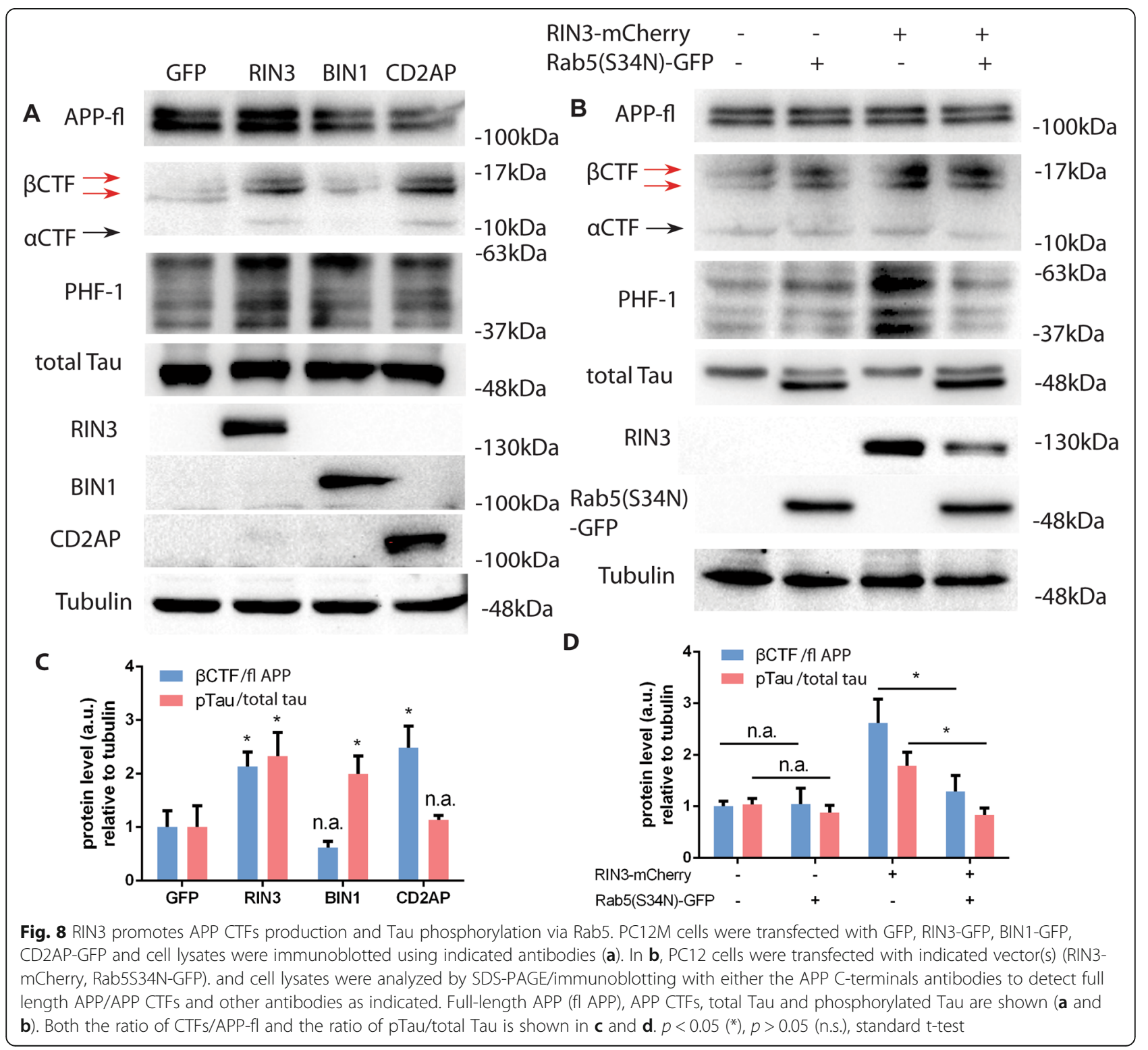


effectively blocked the increase of both $\beta$ CTFs and pTau induced by RIN3 overexpression (Fig. $8 \mathrm{~b}$ and d). Of note, although expression of $\mathrm{Rab} 5{ }^{\mathrm{S} 34 \mathrm{~N}}$ appeared to result in the appearance of a lower molecular species of Tau, expression of Rab5 ${ }^{\mathrm{S} 34 \mathrm{~N}}$ alone did not alter the level of pTau (Fig. 8b). However, we did observe the appearance of a second Tau species with a lower molecular mass in these $\mathrm{Rab} 5^{\mathrm{S} 34 \mathrm{~N}}$-expressing cell samples. To further address the influence of Rab5 $5^{\mathrm{S} 34 \mathrm{~N}}$ on total Tau level, we respectively transfected GFP-Rab5 ${ }^{\mathrm{WT}}$ and GFP-Rab5 ${ }^{\mathrm{S} 34 \mathrm{~N}}$ in PC12 cells using GFP as a control. Total Tau proteins were detected with the same antibody. As shown in Supplementary Figure 5 , it turned out that GFP-Rab5 ${ }^{\mathrm{WT}}$ and GFP-Rab5 ${ }^{\mathrm{S} 34 \mathrm{~N}}$, but not GFP itself, induced the appearance of this lower molecular species of Tau. Therefore, it is likely that overexpression of Rab5 promotes cleavage of Tau. Taken together, these data have demonstrated that the RIN3/BIN1/CD2AP complex may have a dual role in regulating APP processing and Tau phosphorylation; The RIN3/CD2AP arm enhances Rab5 activation and thus to affect APP processing that favors the production and accumulation of neurotoxic $\beta C T F s$, while the RIN3/BIN1 axis may affect Tau phosphorylation.

\section{Discussion}

Although increasing genetic evidence has uncovered RIN3 as an AD risk factor, presently, little is known about its exact role in cellular pathogenesis in either LOAD or EOAD. Here we have demonstrated that RIN3 was significantly upregulated in APP/PS1 mouse model of AD. Enhanced expression of RIN3 correlated with enlarged Rab5 early endosomes in primary cultured E18 BFCN neurons from APP/PS1 mice. We have shown that RIN3 interacted with and recruited BIN1 and CD2AP to Rab5 early endosomes. We further demonstrated that by interacting with CD2AP, RIN3 regulated APP trafficking and processing and increased expression of RIN3/CD2AP enhanced the production of neuronal toxic APP $\beta C T F s$. On the other hand, through binding to BIN1, increased expression of RIN3 enhanced Tau phosphorylation. The effect of increased expression of RIN3 on both the production of $\beta$ CTFs and pTau increase is likely mediated by activating Rab5. Our current study has thus provided novel insights into the cellular and molecular mechanisms by which increased RIN3 expression contributes to cellular and neuronal dysfunction that may contribute importantly to early pathogenesis in $\mathrm{AD}$.

Following the initial discovery of RIN3 association with LOAD in a large scaled GWAS in 2011 [13], genetic variant RIN3 was identified in a prospective cohort study of 74,754 individuals together with BIN1 and CD2AP to be associated with increased risk of Alzheimer's disease, all dementia, and suggested vascular dementia independent of the strong APOE $\varepsilon 4$ allele [54]. A recent study enrolled cognitively healthy centenarians as extreme controls suggested that compared to agematched controls, the effect size increase was significant for RIN3 (4.5-fold), even more significant than APOE$\varepsilon 2$ (2.2-fold) and APOE- $\varepsilon 4(2.0$-fold) [22]. This conclusion indicated that the LOAD-RIN3 variant is highly correlated with cognitive function. In addition, a 2017 GWAS defined a missense mutation in RIN3 (W63C) is associated with sporadic EOAD [23]. Therefore, strong evidence supports a role of RIN3 in AD pathogenesis.

In a gene methylation profiling study of blood and brain samples from $22 \mathrm{AD}$ and 26 normal control subjects (27 males, 21 females), AD samples showed significant group-wide hypomethylation of $7 \mathrm{CpGs}$ located within the 3'UTR of RIN3 (CpG1 p = 0.019, CpG2 $\mathrm{p}=$ 0.018 , CpG3 $\mathrm{p}=0.012$, CpG4 $\mathrm{p}=0.009$, CpG5 $\mathrm{p}=$ 0.002 , CpG6 $\mathrm{p}=0.018$, and CpG7 $\mathrm{p}=0.013$, respectively). The effect was specific since other LOAD risk factors (PTK2 $\beta$, ABCA7, SIRT1, or MEF2C) did not show significant changes in methylation of their promoters [24]. Hypomethylation is believed to result in enhanced expression of the perspective gene [55]. Consistent with these studies, we detected that RIN3 expression was consistently upregulated in APP/PS1 mice even as early as in cultured E18 BFCNs. The increase in RIN3 expression precedes the appearance of both $A \beta$-amyloidcontaining neurotic plaques and phosphorylated Taucontaining neurofibrillary tangles in APP/PS1 mice with the appears after 3 months of age while the latter becomes visible after 4 months of age [56]. Based on these observations, we speculate that upregulation of RIN3 expression is an early event in AD pathogenesis and that RIN3-induced endocytic dysfunction may play a crucial role in the initiation of early cellular and neuronal events that leads to AD pathogenesis.

Our study has also revealed that RIN3 interacted with BIN1 and CD2AP. BIN1 interacts with the N-terminal region (amino acids 1-586) encompassing the $\mathrm{SH} 3$ domain of RIN3 [26]. CD2AP may bind via its SH3 domain to the two PRD domains (367-390aa, 445-462aa) in RIN3 [47]. We confirmed these interactions in our genetic deletion experiments: removal of $\mathrm{SH} 2, \mathrm{RH}, \mathrm{Vps} 9$ or the Ras domain on RIN3 didn't disrupt colocalization of RIN3 with BIN1 and CD2AP. However, without these domains, the RIN3/BIN1/CD2AP complexes failed to be recruited to early endosomes. We thus speculate that RIN3 acts as a scaffold to tether BIN1 and CD2AP specifically to early endosomes.

Amyloidogenic cleavage of APP to yield A $\beta$ likely occurs predominantly in the intracellular compartments [20, 57-59] Under normal conditions, early endosomes marked by Rab5 are a major site of APP processing by $\beta$-secretase to yield $\beta$ CTFs $[18,19]$. $\beta$ CTFs is further 
processed in late endosomes or trans-Golgi network (TGN) to give rise to $A \beta$. The neuronal toxicity of $\beta C T F$ has been observed in multiple studies. Recent studies have pointed to strong adverse impact of $\beta C T F$ on synaptic plasticity and neuronal function and is intimately linked to early cellular pathology, independent of $A \beta$, in AD [14, 18, 21, 53, 60-63]. Axonal APP was more likely to undergo cleavage and produce $A \beta$. Accordingly, we found overexpressed RIN3 in primary cortical neurons impaired APP and BACE1 trafficking by increasing stationary vesicles and slower trafficking velocities, and upregulating RIN3 led to increased APP CTFs production. This effect was rescued by $\mathrm{Rab} 5^{\mathrm{S} 34 \mathrm{~N}}$, supporting that increased RIN3 promoted APP CTFs in early endosomes.

Unlike RIN3, BIN1 and CD2AP have been extensively studied for their function in regulating endocytic trafficking and their role in $\mathrm{AD}$ pathogenesis [20, 51, 52, 64]. CD2AP, a membrane-associated scaffolding protein, likely controls the assembly of protein complexes, participates in endocytosis and endocytic trafficking to transmit intracellular signals [45]. It was shown that CD2AP, by virtue of its multiple protein-protein binding modules, interacts with multiple proteins involved in diverse biological processes [45]. Previous study has shown that suppression of CD2AP resulted in a decreased $A \beta_{42} /$ $\mathrm{A} \beta_{40}$ ratio both in N2a neuron-like cell lines and in 1 month-old APP/PS1 mice [65]. And deletion of CD2AP also decreased APP CTFs [52]. Consistent with these studies, our results show that increased CD2AP compromised APP and BACE1 trafficking, leading to increased APP-CTFs.

BIN1, also known as amphyphisin II, has 10 isoforms. The longest isoforms expressed predominantly in central nerve system contains a unique clathrin-AP2 binding region (CLAP), while the shorter isoforms are ubiquitous [11]. And our data shown that the brain-specific isoform level was decreased in 3 months old APP/PS1 mice, while the ubiquitous isoform level was increased. Although the significance is presently unknown, the changes in expression of BIN1 isoforms have also been recently reported in human postmortem brain samples when comparing healthy control to $\mathrm{AD}$ patients $[11,50$, $64,66,67]$. Although the role of BIN1 in regulating APP processing is currently under debate [51, 52, 68, 69], BIN1 has been found to interact with Tau [51, 64, 68, 69], indicating its potential role in regulating Tau biology. This is confirmed in a recent study that increased BIN1 expression was found disrupting their eye morphology by modulating Tau pathology in Drosophilia rather than $A \beta 42$ pathology [68]. Consistent with these findings, we have demonstrated that RIN3/BIN1 overexpression led to upregulated hyperphosphorylated Tau. Although the role of BIN1 in regulating Rab5 early endosome is unclear, a recent study have showed that conditional knockout of BIN1 also induced enlargement of Rab5 endosomes in mice [70]. It is possible that the loss of BIN1 in this case could alter the balance between RIN3/BIN1 and RIN3/CD2AP that frees more RIN3 to activate Rab5. Nevertheless, future studies are needed to define the exact role of the two RIN3 complexes (RIn3/ CD2AP, RIN3/BIN1) in regulating neuronal functions.

Our current study has pointed to a novel but intriguing role for RIN3 in early AD pathogenesis: By interacting with CD2AP in early endosomes, RIN3 participates in APP trafficking and processing. Increased expression of RIN3 impairs APP trafficking and enhances APP cleavage; By recruiting BIN1 to early endosomes, increased RIN3 expression also promotes Tau hyperphosphorylation. As such, RIN3 contributes importantly to early cellular pathology in AD pathogenesis. Future in vivo studies will be needed to define and validate this novel hypothesis. Since RIN3, BIN1, CD2AP are also expressed in microglial cells [71], it is also vitally important to define the role of RIN3 and the RIN3/ BIN1/CD2AP complexes in processes such as $A \beta$ clearance by microglia in the future as well.

\section{Conclusion}

In summary, we have defined and uncovered an important role for RIN3 in AD pathogenesis. We have shown that increased expression of RIN3 in early stages of $A D$ pathogenesis that results in enlarged Rab5 endosomes. We have also demonstrated that RIN3 recruited two other AD risk factors BIN1 and CD2AP to Rab5 early endosomes. Through Rab5 activation, increased RIN3 and CD2AP impaired trafficking and processing of APP leading to the generation of neuronal toxic APP-CTFs. The increase in the RIN3/BIN1 complex may induce pTau. Together, our study has provided exciting insights into the potential cellular mechanisms by which upregulation of RIN3 contributes to early cellular pathology in early stages of in $\mathrm{AD}$ pathogenesis.

\section{Supplementary information}

Supplementary information accompanies this paper at https://doi.org/10. 1186/s40035-020-00206-1.

Additional file 1: Figure S1. BIN1 and CD2AP colocalize with different RIN3 variant. Different RIN3-GFP constructs were co-transfected into PC12M cells with either CD2AP-flag or BIN1-flag, followed by immunostaining for the flag tagged constructs or Rab5 using specific antibodies. Representative images are shown.

Additional file 2: Figure S2. BIN1 and CD2AP are not recruited to Rab7 late endosome by RIN3. PC12M cells expressing GFP/CD2AP-flag (A, B), RIN3-GFP/CD2AP-flag (C, D), GFP/BIN1-flag (E, F), RIN3-GFP/BIN1-flag (E-H) were immunostained for Rab7 with a specific antibody. Representative images are shown. Colocalization analysis was performed by ImageJ by measuring fluorescence intensity alongside the drawn line. The three different colors peak at the same position If the three proteins were colocalized $(B, D, F, H)$. 
Additional file 3: Figure S3. BIN1 and CD2AP are not recruited to Rab11 recycling endosome by RIN3. PC12M cells expressing GFP/CD2APflag (A, B), RIN3-GFP/CD2AP-flag (C, D), GFP/BIN1-flag (E, F), RIN3-GFP/ BIN1-flag (E-H) were immunostained for Rab11 with a specific antibody. Representative images are shown. Colocalization analysis was performed by ImageJ by measuring fluorescence intensity alongside the drawn line. The three different colors peak at the same position If the three proteins were colocalized $(B, D, F, H)$.

Additional file 4: Figure S4. Colocalization of RIN3, BIN1 and CD2AP with APP. APP-mCherry was co-transfected into mouse E18 primary cortical neurons with either GFP, or RIN3-GFP or BIN1-GFP or CD2AP-GFP (A). Representative images are shown. In $B$, representative images of neurites are shown. C. Results for semiquantitative analyses for colocalization are shown.

Additional file 5: Figure S5. Overexpression of Rab5 induces cleavage of Tau. GFP, GFP-Rab5 ${ }^{W T}$, GFP-Rab5 ${ }^{534 \mathrm{~N}}$ (dominant-negative form) expression vectors were transfected in PC12 cells as indicated. Cells were harvested and lysates were analyzed by SDS-PAGE/ immunoblotting with an antibody against total Tau. GFP antibody was used to detect transfection efficiency and GAPDH was used as endogenous control.

Additional file 6: Table 1. Identify RIN3 interacting protein via Mass Spectrum. Recombinant flag tagged RIN3 protein were purified from HEK293T cells. RIN3-interactomes were identified by Mass Spectrometry. Two replicates were performed. PSM representing abundancy of each protein were used to sort the proteins. BIN1 and CD2AP were highlighted with light yellow in tables.

\section{Abbreviations}

RIN3: Ras And Rab Interactor 3; BIN1: Bridging Integrator 1; CD2AP: Cluster of differentiation 2 Associated Protein; APP: Amyloid precursor protein; PS: Presenilin; NTFs: Neurofibrillary tangles; CTF: Carboxyl terminal fragment; GWAS: Genome wide association study; CLU: Clusterin; CR1: Complement Receptor 1; PICALM: Phosphatidylinositol binding clathrin assembly protein; ABCA7: ATP-binding cassette transporter A7; MS4A4: Membrane-spanning 4domains subfamily $A$; EPHA1: Eph receptor A1; CD33: Cluster of differentiation 33; INPP5D: Inositol polyphosphate-5-phosphatase; MEF2C: Myocyte enhancer factor 2C; HLA-DRB1/HLA-DRB5: the Major Histocompatibility Complex Class II, DRß1 and 5 NME8; ZCWPW1: Zinc finger, CW type with PWWP domain 1; PTK2B: Protein tyrosine kinase 2B; SORL1: Sortilin related receptor L1; CELF1: CUGBP, elav-like family member 1; SLC24A4: Sodium/Potassium/Calcium Exchanger), Member 4;

FERMT2: Fermitin Family Member 2; CASS4: Cas Scaffolding Protein Family Member 4; GEF: Guanine nucleotide exchange factor; LOAD: Late onset AD; EOAD: Early onset AD; sEOAD: Sporadic EOAD; BFCNs: Basal forebrain cholinergic neurons; WT: Wild Type; APOE: Apolipoprotein E; BACE: $\beta$ secretase; TGN: Trans-Golgi network; SH2: Homology 2 (SH2);

TrkA: Tropomyosin receptor kinase A; ChAT: Choline acetyltransferase

\section{Acknowledgements}

We thank Dr. William Mobley for insightful comments. We also thank our colleagues in the Wu lab, Lei Lab and JD lab for assistance, critical. comments, and suggestions. We also thank Dr. B. Head and Dr. S. Wang for their assistance. We specially thank Dr. Xiaojing Lin for GO analysis of MS data.

\section{Authors' contributions}

CW, JD and RS conceived the project and designed the experiments. CW and RS analyzed the data and wrote the paper. RS and LH performed most of the experiments, assisted by XZ, WX, SL, SF, WY, KS, BS and RR. All authors read, approved and commented on the manuscript.

\section{Funding}

The project was financially supported by the National Natural Science Foundation of China [\#81630029 and \#81871005 for JD], the National Key R\&D Program of China [\#2016YFC13060000 for JD]. UCSD ADRC P50 Pilot Grant (Wu).

\section{Availability of data and materials}

The datasets used and/or analyzed during the current study are available from the corresponding author on reasonable request.

\section{Ethics approval and consent to participate}

Not applicable.

\section{Consent for publication}

Not applicable.

\section{Competing interests}

The authors declare that they have no competing interests.

\section{Author details}

${ }^{1}$ Institute of Neurology, Ruijing Hospital, Shanghai JiaoTong University School of Medicine, 197 Ruijin Er Rd., Shanghai 200025, China. ${ }^{2}$ Department of Neurosciences, University of California San Diego School of Medicine, Room 312 MC-0624,9500 Gilman Drive, La Jolla, CA 92093-0624, USA. ${ }^{3}$ Shanghai Institute of Precision Medicine, Shanghai 200125, China. ${ }^{4}$ Department of Neurology, Zhuijiang Hospital, Southern Medical University, Guangzhou, China. ${ }^{5}$ San Diego VA Health System, San Diego, CA, USA.

Received: 18 December 2019 Accepted: 1 June 2020

Published online: 18 June 2020

\section{References}

1. Selkoe DJ, Hardy J. The amyloid hypothesis of Alzheimer's disease at 25 years. EMBO Mol Med. 2016;8(6):595-608.

2. Karch C M, Cruchaga C, Goate A M. Alzheimer's disease genetics: from the bench to the clinic[]]. Neuron. 2014;83(1):11-26.

3. Martínez DB, Soldevilla MAG, Santiago AP, et al. Enfermedad de alzheimer[J]. Medicine-Programa de Formación Médica Continuada Acreditado. 2019; 12(74):4338-46.

4. Karch C M, Goate A M. Alzheimer's disease risk genes and mechanisms of disease pathogenesis[]]. Biol Psychiatry. 2015;77(1):43-51.

5. Doody RS, Aisen PS, Iwatsubo T. Semagacestat for treatment of Alzheimer's disease. N Engl J Med. 2013;369(17):1661.

6. Doody RS, Raman R, Farlow M, Iwatsubo T, Vellas B, Joffe S, et al. A phase 3 trial of Semagacestat for treatment of Alzheimer's disease. N Engl J Med. 2013:369(4):341-50

7. Gauthier S, Feldman HH, Schneider LS, Wilcock GK, Frisoni GB, Hardlund JH, et al. Efficacy and safety of tau-aggregation inhibitor therapy in patients with mild or moderate Alzheimer's disease: a randomised, controlled, double-blind, parallel-arm, phase 3 trial. Lancet. 2016;388(10062):2873-84.

8. Mahley RW, Weisgraber KH, Huang Y. Apolipoprotein E: structure determines function, from atherosclerosis to Alzheimer's disease to AIDS. J Lipid Res. 2009;50 Suppl:S183-8.

9. Gatz M, Reynolds CA, Fratiglioni L, Johansson B, Mortimer JA, Berg S, et al. Role of genes and environments for explaining Alzheimer disease. Arch Gen Psychiatry. 2006;63(2):168-74.

10. Harold D, Abraham R, Hollingworth P, Sims R, Gerrish A, Hamshere ML, et al. Genome-wide association study identifies variants at CLU and PICALM associated with Alzheimer's disease. Nat Genet. 2009:41:1088-93.

11. Chapuis J, Hansmannel F, Gistelinck M, Mounier A, Van Cauwenberghe C, Kolen $\mathrm{KV}$, et al. Increased expression of BIN1 mediates Alzheimer genetic risk by modulating tau pathology. Mol Psychiatry. 2013;18(11):1225-34.

12. Seshadri S, Fitzpatrick AL, Ikram MA, DeStefano AL, Gudnason V, Boada M, et al. Genome-wide analysis of genetic loci associated with Alzheimer disease. JAMA. 2010:303(18):1832-40.

13. Sherva R, Baldwin CT, Inzelberg R, Vardarajan B, Cupples LA, Lunetta $K$, et al. Identification of novel candidate genes for Alzheimer's disease by autozygosity mapping using genome wide SNP data. J Alzheimers Dis. 2011;23(2):349-59.

14. Cataldo AM, Peterhoff CM, Troncoso JC, Gomez-Isla T, Hyman BT, Nixon RA. Endocytic pathway abnormalities precede amyloid beta deposition in sporadic Alzheimer's disease and Down syndrome: differential effects of APOE genotype and presenilin mutations. Am J Pathol. 2000;157:277-86.

15. Ginsberg SD, Mufson EJ, Alldred MJ, Counts SE, Wuu J, Nixon RA, et al. Upregulation of select rab GTPases in cholinergic basal forebrain neurons in mild cognitive impairment and Alzheimer's disease. J Chem Neuroanat. 2011;42(2):102-10 
16. Ginsberg SD, Mufson EJ, Counts SE, Wuu J, Alldred MJ, Nixon RA, et al. Regional selectivity of rab5 and rab7 protein upregulation in mild cognitive impairment and Alzheimer's disease. J Alzheimers Dis. 2010;22(2):631-9.

17. Nixon RA. Endosome function and dysfunction in Alzheimer's disease and other neurodegenerative diseases[J]. Neurobiol Aging. 2005;26(3):373-82.

18. Cataldo AM, Petanceska S, Terio NB, Peterhoff CM, Durham R, Mercken M, et al. $A \beta$ localization in abnormal endosomes: association with earliest $A \beta$ elevations in AD and Down syndrome. Neurobiol Aging. 2004;25(10):126372.

19. Kinoshita A. Demonstration by FRET of BACE interaction with the amyloid precursor protein at the cell surface and in early endosomes. J Cell Sci. 2003; 116(Pt 16):3339-46.

20. Thinakaran $\mathrm{G}, \mathrm{Koo} \mathrm{EH}$. Amyloid precursor protein trafficking, processing, and function[J]. J Biol Chem. 2008;283(44):29615-9.

21. Xu W, Weissmiller AM, White JA 2nd, Fang F, Wang X, Wu Y, et al. Amyloid precursor protein-mediated endocytic pathway disruption induces axonal dysfunction and neurodegeneration. J Clin Invest. 2016;126(5):1815-33.

22. Tesi N, van der Lee SJ, Hulsman M, Jansen IE, Stringa N, van Schoor N, et al. Centenarian controls increase variant effect sizes by an average twofold in an extreme case-extreme control analysis of Alzheimer's disease. Eur J Hum Genet. 2019;27(2):244-53.

23. Kunkle BW, Vardarajan BN, Naj AC, Whitehead PL, Rolati S, Slifer S, et al. Early-onset Alzheimer disease and candidate risk genes involved in Endolysosomal transport. JAMA Neurol. 2017;74(9):1113-22.

24. Boden KA, Barber IS, Clement N, Patel T, Guetta-Baranes T, Brookes KJ, et al. Methylation Profiling RIN3 and MEF2C Identifies Epigenetic Marks Associated with Sporadic Early Onset Alzheimer's Disease. J Alzheimers Dis Rep. 2017;1(1):97-108.

25. Pathak GAS, Talisa K, Sun J, Zhou Z, Daniel AA, Johnson L, O'Bryant S, Phillips NR, Barber RC. Genome-Wide Methylation of Mild Cognitive Impairment in Mexican Americans Highlights Genes Involved in Synaptic Transport, Alzheimer's Disease-Precursor Phenotypes, and Metabolic Morbidities. J Alzheimer's Dis. 2019. Pre-press. https://doi.org/10.3233/JAD-190634.

26. Kajiho H. RIN3: a novel Rab5 GEF interacting with amphiphysin II involved in the early endocytic pathway. J Cell Sci. 2003;116:4159-68.

27. Kajiho H, Sakurai K, Minoda T, Yoshikawa M, Nakagawa S, Fukushima S, et al Characterization of RIN3 as a guanine nucleotide exchange factor for the Rab5 subfamily GTPase Rab31. Biol Chem. 2011;286(27):24364-73.

28. Grbovic OM, Mathews PM, Jiang Y, Schmidt SD, Dinakar R, Summers-Terio $\mathrm{NB}$, et al. Rab5-stimulated up-regulation of the endocytic pathway increases intracellular $\beta$-cleaved amyloid precursor protein carboxyl-terminal fragment levels and A $\beta$ production. J Biol Chem. 2003;278(33):31261-8.

29. Bucci C, Parton R G, Mather I H, et al. The small GTPase rab5 functions as a regulatory factor in the early endocytic pathway[J]. Cell. 1992;70(5):715-28.

30. Gorvel JP, Chavrier P, Zerial M, Gruenberg J. rab5 controls early endosome fusion in vitro. Cell. 1991;64(5):915-2.

31. Schnitzler AC, Lopez-Coviella I, Blusztajn JK. Purification and culture of nerve growth factor receptor (p75)-expressing basal forebrain cholinergic neurons. Nat Protoc. 2008;3(1):34-40.

32. Sung $K$, Ferrari LF, Yang W, Chung C, Zhao X, Gu Y, et al. Swedish nerve growth factor mutation (NGF(R100W)) defines a role for TrkA and p75(NTR) in nociception. J Neurosci. 2018;38(14):3394-413.

33. Brückner A, Polge C, Lentze $N$, et al. Yeast two-hybrid, a powerful tool for systems biology[J]. Int J Mol Sci. 2009;10(6):2763-88.

34. Fang F, Yang W, Florio JB, Rockenstein E, Spencer B, Orain XM, et al. Synuclein impairs trafficking and signaling of BDNF in a mouse model of Parkinson's disease. Sci Rep. 2017;7(1):3868.

35. Garcia-Alloza M, Robbins EM, Zhang-Nunes SX, Purcell SM, Betensky RA, Raju $S$, et al. Characterization of amyloid deposition in the APPswe/PS1dE9 mouse model of Alzheimer disease. Neurobiol Dis. 2006;24(3):516-24.

36. Jankowsky JL, Fadale DJ, Anderson J, Xu GM, Gonzales V, Jenkins NA, et al. Mutant presenilins specifically elevate the levels of the 42 residue betaamyloid peptide in vivo: evidence for augmentation of a 42 -specific gamma secretase. Hum Mol Genet. 2004:13(2):159-70.

37. Lalonde R, Kim HD, Maxwell JA, Fukuchi K. Exploratory activity and spatial learning in 12-month-old APP(695)SWE/Co+PS1/DeltaE9 mice with amyloid plaques. Neurosci Lett. 2005;390(2):87-92.

38. Wu H, Williams J, Nathans J. Complete morphologies of basal forebrain cholinergic neurons in the mouse. Elife. 2014;3:e02444.
39. Chen XQ, Mobley WC. Exploring the pathogenesis of Alzheimer disease in basal forebrain cholinergic neurons: converging insights from alternative hypotheses. Front Neurosci. 2019;13:446.

40. Fahnestock M, Shekari A. ProNGF and Neurodegeneration in Alzheimer's Disease. Front Neurosci. 2019;13:129.

41. Grothe M, Heinsen $H$, Teipel S. Longitudinal measures of cholinergic forebrain atrophy in the transition from healthy aging to Alzheimer's disease. Neurobiol Aging. 2013;34(4):1210-20.

42. Salehi A, Delcroix JD, Swaab DF. Alzheimer's disease and NGF signaling. J Neural Transm (Vienna). 2004;111(3):323-45.

43. Schliebs R, Arendt T. The cholinergic system in aging and neuronal degeneration. Behav Brain Res. 2011;221(2):555-63.

44. Xie Y, Meeker RB, Massa SM, Longo FM. Modulation of the p75 neurotrophin receptor suppresses age-related basal forebrain cholinergic neuron degeneration. Sci Rep. 2019;9(1):5273.

45. Harrison BJ, Venkat G, Lamb JL, Hutson TH, Drury C, Rau KK, et al. The adaptor protein CD2AP is a coordinator of Neurotrophin signaling-mediated axon arbor plasticity. J Neurosci. 2016;36(15):4259.

46. Balaji K, Mooser C, Janson CM, Bliss JM, Hojjat H, Colicelli J. RIN1 orchestrates the activation of RAB5 GTPases and ABL tyrosine kinases to determine the fate of EGFR. J Cell Sci. 2012;125(Pt 23):5887-96.

47. Rouka E, Simister PC, Janning M, Kumbrink J, Konstantinou T, Muniz JRC, et al. Differential recognition preferences of the three Src homology 3 (SH3) domains from the adaptor CD2-associated protein (CD2AP) and direct association with Ras and Rab Interactor 3 (RIN3). J Biol Chem. 2015;290(42): 25275-92.

48. Almeida CG, Mirfakhar FS, Perdigão C, et al. Impact of late-onset Alzheimer's genetic risk factors on beta-amyloid endocytic production[J]. Cell Mol Life Sci. 2018;75(14):2577-89.

49. Karch CM, Jeng AT, Nowotny P, Cady J, Cruchaga C, Goate AM. Expression of Novel Alzheimer's Disease Risk Genes in Control and Alzheimer's Disease Brains. PLoS One (IF: 2.776). 2012;7(11):e50976.

50. Holler CJ, Davis PR, Beckett TL, Platt TL, Webb RL, Head E, et al. Bridging integrator 1 (BIN1) protein expression increases in the alzheimer's disease brain and correlates with neurofibrillary tangle pathology. J Alzheimers Dis. 2014; 42(4):1221-7.

51. Andrew RJ, De Rossi $P$, Nguyen $P$, Kowalski HR, Recupero AJ, Guerbette T, et al. Reduction of the expression of the late-onset Alzheimer's disease (AD) risk-factor BIN1 does not affect amyloid pathology in an AD mouse model. J Biol Chem. 2019;294(12):4477-87.

52. Ubelmann F, Burrinha T, Salavessa L, Gomes R, Ferreira C, Moreno N, et al. Bin1 and CD2AP polarise the endocytic generation of beta-amyloid. EMBO Rep. 2017;18(1):102-22.

53. Kwart D, Gregg A, Scheckel C, Murphy E, Paquet D, Duffield M, et al. A large panel of isogenic APP and PSEN1 mutant human iPSC neurons reveals shared Endosomal abnormalities mediated by APP beta-CTFs, Not Abeta. Neuron. 2019;104(2):256-270.e5.

54. Juul Rasmussen I, Tybjærg-Hansen A, Rasmussen KL, Nordestgaard BG, Frikke-Schmidt R. Blood-brain barrier transcytosis genes, risk of dementia and stroke: a prospective cohort study of 74,754 individuals. Eur $J$ Epidemiol. 2019;34(6):579-90.

55. Bogdanović O, Lister R. DNA methylation and the preservation of cell identity[J]. Curr Opin Genet Dev. 2017:46:9-14.

56. Blanchard V, Moussaoui S, Czech C, Touchet N, Bonici B, Planche M, et al. Time sequence of maturation of dystrophic neurites associated with $A \beta$ deposits in APP/PS1 transgenic mice. Exp Neurol. 2003;184(1):247-63.

57. Toh WH, Gleeson PA. Dysregulation of intracellular trafficking and endosomal sorting in Alzheimer's disease: controversies and unanswered questions. Biochem J. 2016;473(14):1977-93.

58. Spencer B, Desplats P A, Overk C R, et al. Reducing endogenous a-synuclein mitigates the degeneration of selective neuronal populations in an Alzheimer's disease transgenic mouse model[J]. J Neurosci. 2016;36(30): 7971-84.

59. Koo EH, Squazzo SL. Evidence that production and release of amyloid ??-protein involves the endocytic pathway. J Biol Chem. 1994.

60. Laifenfeld D, Patzek $\amalg$, McPhie DL, Chen Y, Levites Y, Cataldo AM, et al. Rab5 mediates an amyloid precursor protein signaling pathway that leads to apoptosis. J Neurosci. 2007;27(27):7141-53.

61. Lauritzen I, Pardossi-Piquard R, Bauer C, Brigham E, Abraham JD, Ranaldi S, et al. The beta-secretase-derived C-terminal fragment of betaAPP, C99, but 
not Abeta, is a key contributor to early intraneuronal lesions in tripletransgenic mouse hippocampus. J Neurosci. 2012;32(46):16243-55a.

62. Lauritzen I, Pardossi-Piquard R, Bourgeois A, Pagnotta S, Biferi MG, Barkats M, et al. Intraneuronal aggregation of the beta-CTF fragment of APP (C99) induces Abeta-independent lysosomal-autophagic pathology. Acta Neuropathol. 2016;132(2):257-76.

63. De Rossi P, Andrew RJ, Musial TF, Buggia-Prevot V, Xu G, Ponnusamy M, et al. Aberrant accrual of BIN1 near Alzheimer's disease amyloid deposits in transgenic models. Brain Pathol. 2019;29(4):485-501.

64. De Rossi P, Buggia-Prévot V, Clayton BLL, Vasquez JB, Van Sanford C, Andrew RJ, et al. Predominant expression of Alzheimer's disease-associated BIN1 in mature oligodendrocytes and localization to white matter tracts. Mol Neurodegener. 2016;11(1):59.

65. Liao F, Jiang H, Srivatsan S, Xiao Q, Lefton KB, Yamada K, et al. Effects of CD2-associated protein deficiency on amyloid- $\beta$ in neuroblastoma cells and in an APP transgenic mouse model. Mol Neurodegener. 2015;10:12.

66. Malki I, Cantrelle FX, Sottejeau Y, Lippens G, Lambert JC, Landrieu I. Regulation of the interaction between the neuronal BIN1 isoform 1 and tau proteins - role of the SH3 domain. FEBS J. 2017;284(19):3218-29.

67. Sottejeau Y, Bretteville A, Cantrelle FX, Malmanche N, Demiaute F, Mendes T, et al. Tau phosphorylation regulates the interaction between BIN1's SH3 domain and Tau's proline-rich domain. Acta Neuropathol Commun. 2015;3:58.

68. Calafate S, Flavin W, Verstreken P, Moechars D. Loss of Bin 1 promotes the propagation of tau pathology. Cell Rep. 2016;17(4):931-40.

69. Crotti A, Sait HR, McAvoy KM, Estrada K, Ergun A, Szak S, et al. BIN1 favors the spreading of tau via extracellular vesicles. Sci Rep. 2019:9(1):9477.

70. De Rossi P, Nomura T, Andrew RJ, Masse NY, Sampathkumar V, Musial TF, et al. Neuronal BIN1 regulates presynaptic neurotransmitter release and memory consolidation. Cell Rep. 2020;30(10):3520-35 e7.

71. Hansen DV, Hanson JE, Sheng M. Microglia in Alzheimer's disease. J Cell Biol. 2018;217(2):459

Ready to submit your research? Choose BMC and benefit from:

- fast, convenient online submission

- thorough peer review by experienced researchers in your field

- rapid publication on acceptance

- support for research data, including large and complex data types

- gold Open Access which fosters wider collaboration and increased citations

- maximum visibility for your research: over $100 \mathrm{M}$ website views per year

At $\mathrm{BMC}$, research is always in progress.

Learn more biomedcentral.com/submissions 